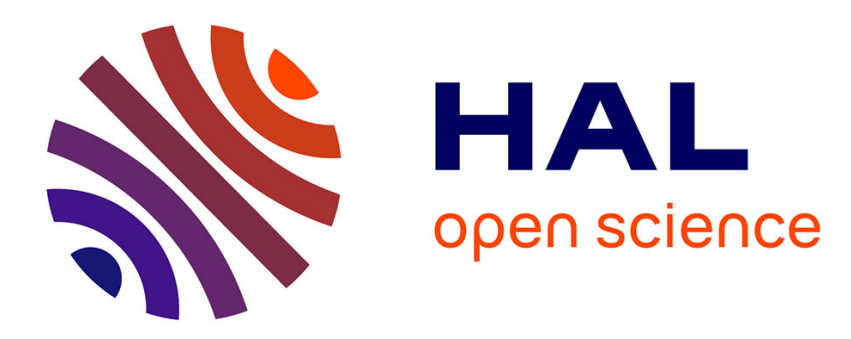

\title{
Les classifications des sciences mathématiques en Grèce ancienne
}

Bernard Vitrac

\section{To cite this version:}

Bernard Vitrac. Les classifications des sciences mathématiques en Grèce ancienne. Archives de Philosophie, 2005, 68, pp.269-301. hal-00175169

\section{HAL Id: hal-00175169 \\ https://hal.science/hal-00175169}

Submitted on 2 Feb 2010

HAL is a multi-disciplinary open access archive for the deposit and dissemination of scientific research documents, whether they are published or not. The documents may come from teaching and research institutions in France or abroad, or from public or private research centers.
L'archive ouverte pluridisciplinaire HAL, est destinée au dépôt et à la diffusion de documents scientifiques de niveau recherche, publiés ou non, émanant des établissements d'enseignement et de recherche français ou étrangers, des laboratoires publics ou privés. 


\section{Les classifications des sciences mathématiques en Grèce ancienne ${ }^{1}$}

Bernard Vitrac, CNRS, UMR 8567, Centre Louis Gernet, Paris

«Réponse en vérité vaillante et libérale!

On te demande une seule chose, et tu en donnes une quantité,

et du bigarré à la place de l'uni ».

Platon, Théétète, $146 \mathrm{~d} 3-4^{2}$.

La classification des sciences mathématiques dans l'Antiquité gréco-romaine peut s'envisager de bien des manières :

- comme une portion d'un inventaire raisonné et systématique de l'ensemble des connaissances humaines (de l'époque);

- comme la mise en ordre d'un processus progressif de découvertes ou d'inventions, considéré comme temporairement (définitivement ?) stabilisé.

- comme le fondement d'un programme éducatif énumérant les savoirs que telle ou telle partie de la communauté politique doit maîtriser pour remplir correctement le rôle social qui est le sien.

- comme un outil utile au bibliothécaire pour ranger une collection d'ouvrages mathématiques.

A chaque fois il s'agit de découvrir ou d'imposer un ordre, en cherchant à satisfaire deux exigences entre lesquelles une certaine tension est perceptible : d'une part discriminer, répartir, et donc séparer, d'autre part identifier des relations voire, quand la perspective est génétique, des filiations ou une progression. Et donc articuler un Tout en ses parties, problème éminemment philosophique, notamment pour un Platonicien. Il ne manque pas de métaphore pour porter la première exigence - l'aspect statique de la classification - , le plus facile à capturer : division du territoire (du savoir), arbre (des sciences), tableau (systématique des connaissances humaines) ... Pour qu'un tel schéma ne se réduise pas à une juxtaposition arbitraire, il faut que les propriétés qui caractérisent les classes s'attribuent de manière essentielle et stable aux individus considérés, qu'elles mettent en évidence des relations entre lesdites classes. S'il s'agit d'une matière historiquement déterminée, elles devront prévoir des lieux où insérer des éléments à venir.

Dans le cas des sciences mathématiques anciennes, un tel programme revient donc à se demander : « qu'est-ce que le (ou la) mathématique et quelles espèces, sous-espèces peut-on y distinguer ? », «En quoi réside leur(s) élément(s) commun(s) et quel(s) critère(s) les différencie(nt) ? ». Du point de vue historique qui sera le mien ici, « quand et dans quel(s) contexte(s) ce genre de questionnement est-il apparu et quels en étaient les promoteurs ? ». Nous verrons qu'il s'agit pour l'essentiel de philosophes. Il convient donc de se demander également quels rapports il y avait entre pratiques mathématiques et tentatives de classification.

\footnotetext{
1 J'ai présenté des versions préliminaires de ce texte dans différents séminaires et dans le cours que j'ai donné en 2002-2003 dans le cadre de la formation en philosophie ancienne du Centre d'Études Anciennes de l'École Normale Supérieure, rue d'Ulm. Je remercie tou(te)s les participant(e)s à ces stimulantes réunions pour leurs remarques et critiques.

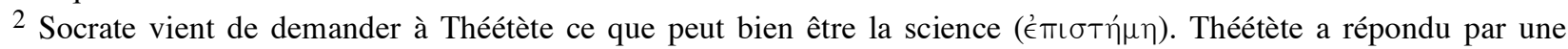
énumération. Pour les traductions de Platon, j'utilise celle de L. Robin et J. Moreau, Bibliothèque de la Pléiade. Paris, Gallimard, 1950. Ici, vol. II, p. 89.
} 
La première question consiste, en suivant le modèle de Socrate dans le Théétète, à s'interroger sur ce que peut bien être «la mathématique ». Même en français le singulier heurte l'usage ordinaire qui voudrait que les mathématiques soient plurielles. Le singulier est associé à un point de vue philosophique ou paradoxal, par exemple bourbakiste. La contribution ancienne à la définition de la mathématique est plutôt décevante, à l'exception notable d'Aristote. Pour pallier ce déficit, les autres - c'est le cas de Platon - optent souvent pour l'énumération. Ce sera mon premier point. Un seul auteur ancien, Proclus de Lycie ( $\mathrm{V}^{\mathrm{e}}$ siècle), présente explicitement des énumérations - de fait deux - décrites comme des classifications des sciences mathématiques, dans le prologue de son Commentaire au premier Livre des Éléments d'Euclide. Il s'agit de deux manières de diviser la mathématique entière, ou le savoir mathématique global ( $\dot{\eta}$

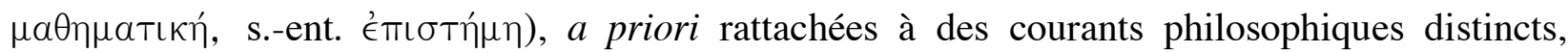
puisque le premier schéma, en quatre sciences (arithmétique, musique, géométrie, sphérique), est décrit comme pythagoricien, tandis que le second, qui comporte huit branches principales (et de nombreuses sous-espèces) - arithmétique, géométrie, mécanique, astronomie, optique, géodésie, canonique et harmonique - , est rapportée sous l'autorité du Stoïcien Géminus de Rhodes (probablement $\mathrm{I}^{\mathrm{e}} \mathrm{s}$. avant notre ère) ${ }^{3}$. J'examinerai donc ce (double) témoignage, tout particulièrement en ce qui concerne le schéma géminien, peu documenté par ailleurs.

Il est en effet facile de voir que l'une des sources essentielles de Proclus en ce qui concerne le système pythagoricien est Nicomaque de Gérase (début II ${ }^{\mathrm{e}} \mathrm{s}$.). La discussion remonte donc (au moins) à la charnière des époques hellénistique et impériale dans un contexte intellectuel où s'épanouissent les pratiques érudites, notamment les commentaires. Mais il est également très clair que le point de départ de ces tentatives est bien antérieur et réside dans la réflexion historico-anthropologique des Grecs concernant l'origine de la civilisation et le développement progressif des TéXval. Cette réflexion, inaugurée au Ves., est développée par les philosophes du $\mathrm{IV}^{\mathrm{e}} \mathrm{s}$., tout particulièrement Platon et Aristote. J'essaierai de montrer que les programmes éducatifs élaborés par Platon ont joué un rôle essentiel dans la fixation du quadrivium dit (peut-être à tort) pythagoricien et que les grandes lignes de la classification dite de Géminus sont aussi anciennes que l'Académie et Aristote. Celles-ci correspondent assez bien aux découpages que les mathématiciens des époques hellénistique et impériale respectent lorsqu'ils rédigent leurs ouvrages et semblent donc acceptées comme cadre général. La corrélation, souvent établie par les Modernes, entre quadrivium pythagoricien et mathématiques de l'époque classique d'une part, classification de Géminus et sciences hellénistiques d'autre part, est certainement à rejeter. En conclusion je reviendrai brièvement sur l'Antiquité tardive et la postérité de nos deux schémas.

\footnotetext{
${ }^{3}$ V. Procl., In Eucl. I, ed. Friedlein, respectivement p. 35, 1. 17-p. 38, 1. 2 et p. 38, 1. 2-p. 42, 1. 8.
} 
L'énumération des spécialités mathématiques fournit une description de la discipline envisagée globalement par extension, mais une définition par compréhension pourrait s'avérer utile, notamment si l'on concevait des classifications ouvertes, susceptibles d'accueillir de nouvelles spécialités. Par exemple, puisque la classification dite de Géminus contient les quatre disciplines du schéma pythagoricien - malgré quelques fluctuations au niveau des désignations

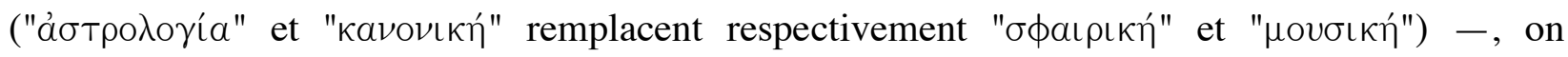
pourrait se demander si la première représente une extension historique de la seconde, comme le présupposent assez naturellement les Modernes. Proclus ne le dit pas et présente ces deux schémas comme des systèmes fermés (et incompatibles). Au demeurant le Diadoque ne semble pas adhérer totalement à la conception "partitionnelle" et relativement statique qui s'exprime dans ces classifications. Dans ce qui précède de son prologue, il a évoqué, à de multiples reprises, un

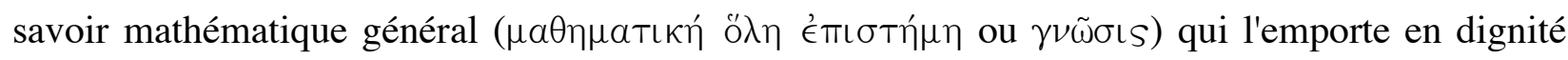
sur les disciplines particulières, comme l'arithmétique et la géométrie, ainsi que l'existence d'une

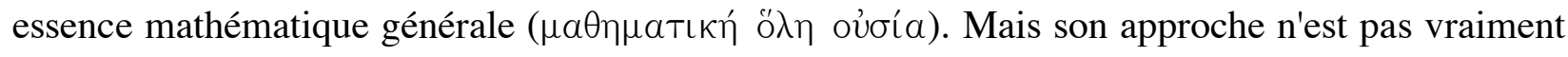

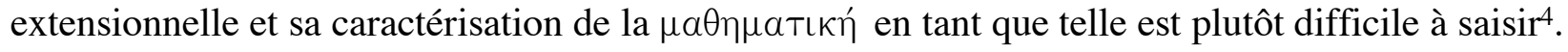

Pour exprimer cette instance particulière de la dualité « Tout / Parties » le grec, depuis le

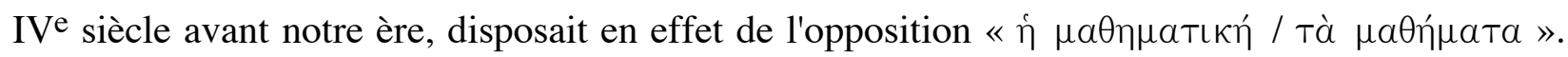

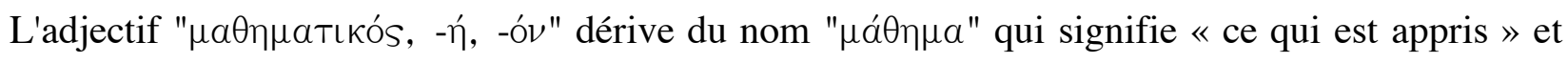
que l'on rattache lui-même au verbe " $\mu \alpha \nu \theta \alpha ́ \nu \omega "$ (apprendre). Ce substantif, au pluriel $(\mu \alpha \theta \eta \dot{\mu} \alpha \tau \alpha)$, acquiert le sens plus circonscrit de «sciences mathématiques » dont on rapporte souvent la fixation aux analyses de Platon et d'Aristote ${ }^{5}$. Mais chez le premier, " $\mu a ́ \theta \eta \mu \alpha "$ garde un sens assez large de «savoir(s) », même lorsqu'il est appliqué à ce que nous appellerions une « science mathématique », comme dans le célèbre passage de la République, Livre VII, 521c-

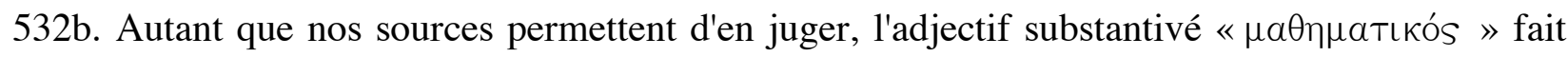
son apparition à l'époque des dialogues dits de maturité, mais ses occurrences sont rares, de fait deux seulement. L'une et l'autre interviennent dans le cadre de distinctions assez larges :

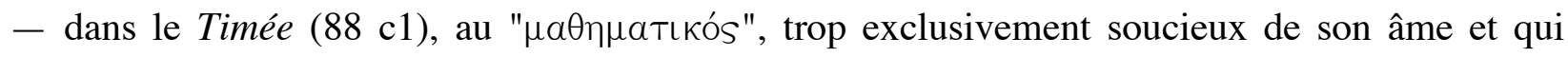
devrait cultiver la gymnastique, Platon oppose celui qui, à l'inverse, ne se préoccupe que de son corps et devrait exercer son âme par « la musique et la philosophie tout entière ». Si la première catégorie peut inclure le mathématicien, cette occurrence renvoie plus généralement à l'intellectuel.

- dans le Sophiste (219 c2), la dimension classificatoire est plus évidente. Il s'agit de distinguer

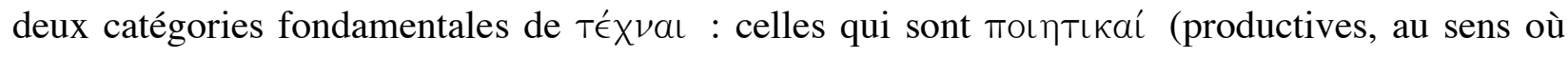
elles impliquent la venue à l'être de quelque chose qui n'existait pas encore) et celles qui sont

\footnotetext{
${ }^{4}$ Réf. à l'article de D. Rabouin à paraitre dans ce même numéro des Archives.

${ }^{5}$ V. par exemple Chantraine, P., dictionnaire étymologique de la langue grecque. 2 vol. Paris, Klincksieck, 1968, p. $664(\mu \alpha \nu \theta a ́ v \omega)$.
} 


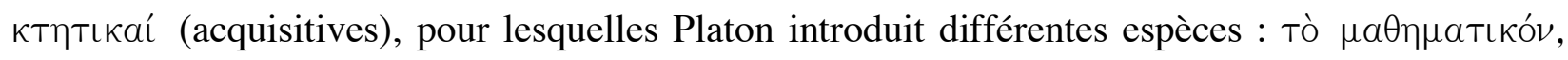

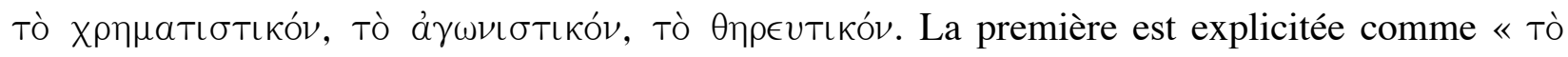
$\tau \tilde{\eta} S \gamma \nu \omega \rho i ́ \sigma \epsilon \omega S$ (s.-ent. $\epsilon \hat{\imath} \delta o s) »$, ce qui concerne la connaissance.

Ni l'une ni l'autre de ces deux occurrences ne visent donc clairement la pleine reconnaissance d'un savoir spécialisé nettement circonscrit. Mes investigations n'ont peut-être pas été complètes, mais je n'ai pas non plus trouvé une définition platonicienne de la mathématique. Qu'il s'agisse de décrire l'activité de certains savants (Hippias d'Élis, Théodore de Cyrène) ou d'élaborer des programmes pédagogiques, le maître de l'Académie se contente de l'énumération,

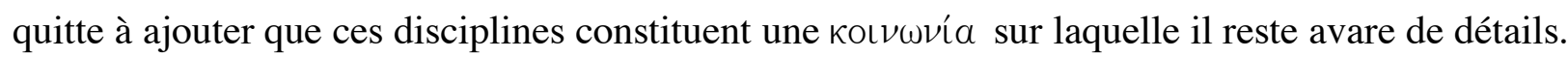

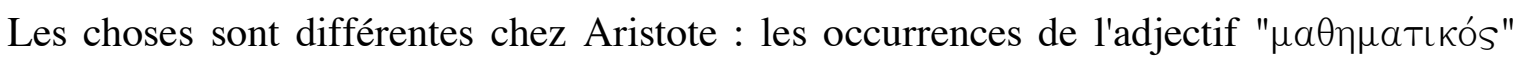
sont chez lui assez nombreuses pour désigner le mathématicien ${ }^{6}$ (o $\left.\mu a \theta \eta \mu a \tau \iota k o ́ s\right)$, souvent par

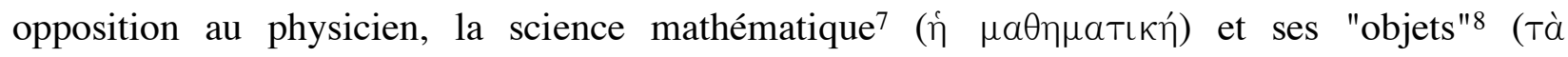
$\mu a \theta \eta \mu а т \iota к a ́)$. Le premier chapitre de Métaphysique E, très important pour notre propos, comporte une dimension partiellement classificatoire. Présupposant une première distinction de la pensée en : «théorétique - pratique - poïétique », Aristote différencie alors trois sortes d'êtres, déterminées en fonction de deux oppositions polaires : 1. Selon qu'ils sont soumis au mouvement et au changement ou, à l'inverse, immuables; 2. Selon qu'ils ont ou non une existence séparée. D'où l'affirmation qu'il existe (au moins) trois sciences (ou "philosophies") théorétiques distinctes : la physique, la mathématique et la science première ou théologie. L'identification qu'il propose pour la mathématique (í $\mu \alpha \theta \eta \mu \alpha \tau \iota \kappa \eta ́)$, ainsi qu'il le reconnaît lui-même, n'est pas évidente mais vaut certainement pour une partie des $\mu a \theta \eta \dot{\mu a t a: ~}$

« Mais la mathématique aussi est théorétique; toutefois, qu'elle soit science d'êtres

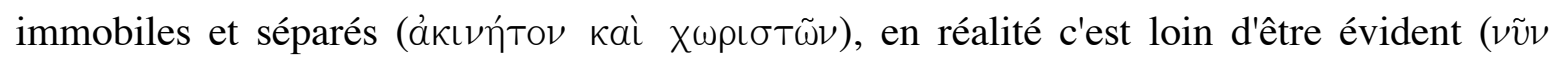

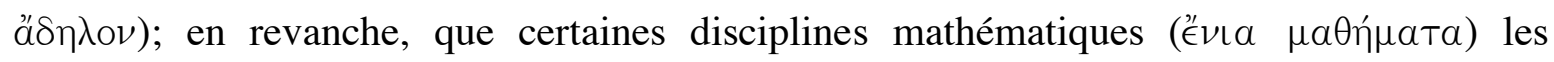

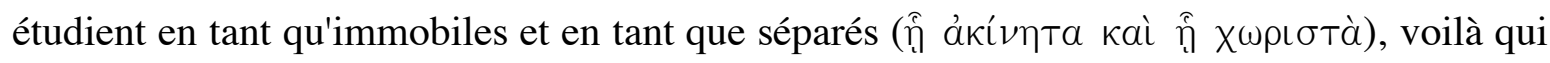
est évident $»^{9}$.

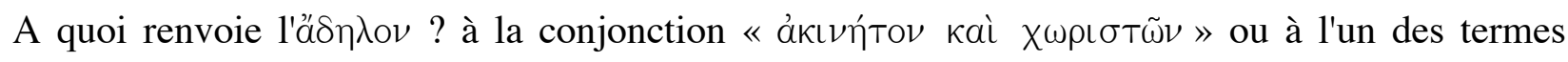
seulement, en l'occurrence " $\chi \omega \rho \iota \sigma \tau \tilde{\omega} \nu "$ ? Dans les deux cas il faut comprendre qu'il y a une différence entre le statut réel des objets mathématiques et la façon dont certaines de ces spécialités les considèrent. Mais si l'on fait la première lecture, cela veut dire qu'en réalité les objets mathématiques ou bien ne sont pas immuables ou bien n'ont pas d'existence séparée. Si

\footnotetext{
6 V. par exemple Phys., II, 2, 193 b25, b31; Met., M, 2, 1077 a9-0.

7 V. Met., E, 1, 1026 a7-32; Met., M, 2, 1076 b33-34.

8 V. par exemple De calo, III, 1, 299 a11-17; Phys., II, 2, 194 a1;Met., A, 6, 987 b15, b28-29; Met., K, 1, 1059 a3821; Met., M, 1, 1076 a16-26; De l'âme, I, 1, 403 b8-16; III, 7, 431 b12-16. Sont notamment qualifiés de "mathématiques", les nombres (Met., M, 1, 1076 a20, M, 2, b39), les lignes (Phys., II, 2, 194 a12) les solides (Met., M, 2, 1076 b17, 23-24, 31), les grandeurs (Met., M, 2, 1077 a21).

${ }^{9}$ Met., E, 1, 1026 a7-10.
} 
l'on admet la seconde, ils sont, ainsi que cela est affirmé un peu plus loin ${ }^{10}$, immobiles mais sans existence séparée. Avec la première interprétation, on ne peut pas identifier les disciplines mathématiques auxquelles il est fait allusion ici11; avec la seconde, il s'agit très certainement de l'arithmétique et de la géométrie. Quoi qu'il en soit, la restriction aristotélicienne ( la difficulté de caractériser globalement les mathématiques telles qu'on les identifiait à l'époque du Stagirite.

Dire que les objets mathématiques se caractérisent par leur statut ontologique en tant qu'immobiles, ne possédant pas d'existence propre mais seulement dérivée, introduit, sinon une définition, du moins une délimitation, de la mathématique et de ses éventuelles prétentions, en la distinguant des deux autres sciences théorétiques. L'intérêt d'Aristote pour une telle entreprise est évident d'autant que le contraste sert aussi à justifier l'existence de la philosophie première. Reste que sa solution ne saurait satisfaire un Platonicien. Cela dit, les philosophes de l'Académie avaient peut-être envisagé une sortie du même genre : caractériser les objets mathématiques par un statut ontologique particulier, intermédiaire entre les choses sensibles et les formes

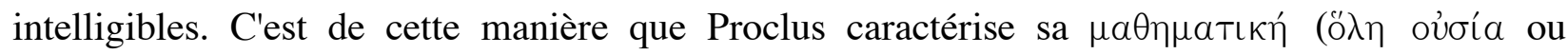

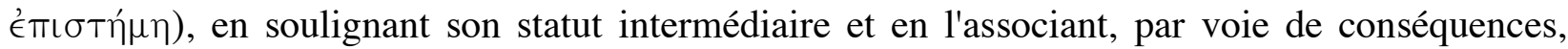
avec la pensée discursive ( $\delta\llcorner a ́ v o \iota \alpha)$. Ce point de vue n'est pas véritablement exprimé dans les dialogues $^{12}$, mais si l'on en croit le Stagirite, l'initiative en reviendrait pourtant à Platon ${ }^{13}$. Comme cela vaut aussi pour les sciences mathématiques particulières, cela ne nous renseigne guère sur leurs relations mutuelles.

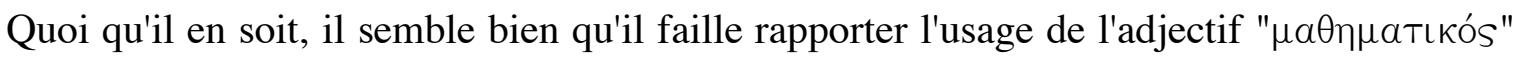
aux discussions de l'Académie puis du Lycée ${ }^{14}$. Que l'on ait alors envisagé un point de vue

\footnotetext{
10 Ibid., 1026 a14-15. La divergence apparente entre les deux passages ( $\chi \omega \rho \iota \sigma T \grave{a} /$ oủ $\chi \omega \rho\llcorner\sigma T a ̀)$ tient à ce que "séparé" peut se dire soit du mode d'existence des objets de science, soit du mode de leur étude («en tant que séparé »), qui peuvent être différents.

${ }^{11}$ M. Crubellier et P. Pellegrin (Aristote, le philosophe et les savoirs, Paris, Seuil, 2002, p. 218) penchent pour cette première lecture et en déduisent que les objets mathématiques auxquels il est fait référence ici ne seraient (en réalité) ni immobiles ni n'auraient d'existence séparée et que, par conséquent, les $\mu a \theta n ́ \mu a \tau a$ dont il est question sont « les plus physiques des sciences mathématiques ». Cela me paraît peu probable, d'une part parce que la négation d'une conjonction est une disjonction (ce qui rend l'identification délicate), d'autre part parce que l'astronomie, qui pour Aristote fait incontestablement partie des parties les plus physiques des mathématiques, ne traite pas ses objets comme immobiles. Enfin, si l'on admet la cohérence des deux assertions (1026 a7-10; a14-15), l'incertitude indiquée

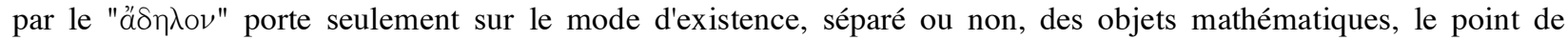
désaccord fondamental entre Aristote et les Platoniciens, et non sur la conjonction des deux critères.

12 V. le commentaire dans D. Ross, Aristotle's Metaphysics. Oxford, Clarendon Press, 1924 (réimpr. Oxford University Press, 1975), pp. 166-168. Il souligne que cette conception ne se trouve pas dans le célébrissime passage de la ligne, en Resp. VI, 509d-511e, mais qu'elle apparaît peut-être en Timée, 50 c.

13 V. Met., A, 6, 987 b14; ibid., Z, 1028 b19.

14 Par ailleurs il existe un certain nombre de témoignages, en particulier chez Diogène Laërce et Jamblique, sur des

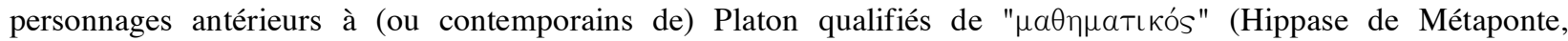
Hippocrate de Chio, Théodore de Cyrène, Archytas de Tarente, Démocrite d'Abdère) ou d'ouvrages, qualifiés de (ou

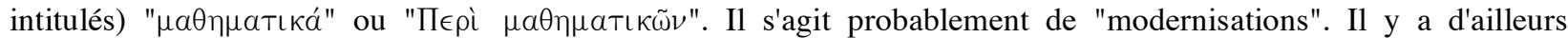
quelques flottements. Ainsi, pour l'ouvrage d'Archytas dont est censé provenir le fragment DK 47 B1, Jamblique

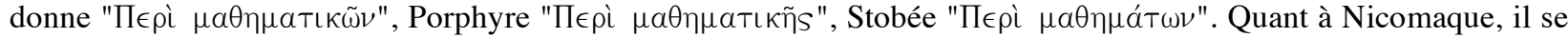

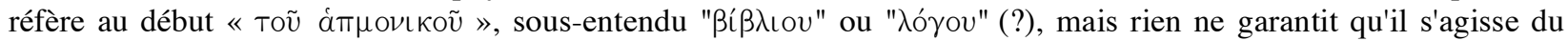

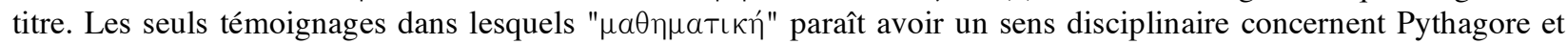
Démocrite. Une raison supplémentaire de douter de leur acribie.
} 
général, en particulier logique et philosophique, sur les mathématiques n'est pas douteux. Aristote ne s'en prive pas. Qu'on ait cherché à caractériser une «mathématique générale », en tant que discipline constituée, est moins sûr. C'était en effet difficile dans le contexte des mathématiques anciennes, compte tenu de la manière dont on avait l'habitude de caractériser une Té $\chi \nu \eta$ par son objet ( $\tau \grave{a} \pi \epsilon \rho \grave{i} \tau \iota^{\prime}$ ), et ce au moins depuis Platon. En raisonnant de manière "ensembliste", c'està-dire par intersection, le seul candidat possible aurait été la théorie des relations, soit sous sa forme semble-t-il ancienne de «théorie des médiétés », soit sous celle que l'on trouve chez Euclide de «théorie des proportions ». L'une et l'autre s'appliquaient aussi bien aux nombres qu'aux grandeurs géométriques. La démarche avait cependant quelque chose de paradoxal dans la mesure où les mathématiques et certaines analyses logico-philosophiques distinguaient précisément objets et relations. On comprend, au moins en partie, le fréquent et commode recours aux classifications.

\section{II}

Revenons aux deux schémas rapportés par Proclus en commençant par le second. Géminus de Rhodes est cité par Alexandre d'Aphrodise qui se réfère à son Abrégé des Météorologiques de Posidonius. Ceci est rapporté par Simplicius ${ }^{15}$. Son activité se plaçait donc entre le premier siècle avant notre ère et le deuxième après. Selon Germaine Aujac, il aurait appartenu, dans sa jeunesse, au cercle des érudits grecs et romains qui entouraient Pompée, et aurait donc été actif dans la seconde moitié du premier siècle avant notre ère ${ }^{16}$. On lui connaît trois ouvrages et les citations que fait Proclus proviennent certainement du troisième, une encyclopédie des mathématiques à caractère historique, mentionnée par Eutocius ${ }^{17}$ sous le titre

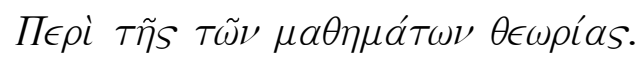

Le principe qui gouverne la classification de Géminus ${ }^{18}$ est l'opposition entre les mathématiques qui traitent des intelligibles seulement (arithmétique et géométrie) et celles qui traitent (en outre ?) des choses sensibles (mécanique, astronomie, optique, géodésie, canonique et logistique). Cette opposition est certainement à rattacher à la distinction ontologique

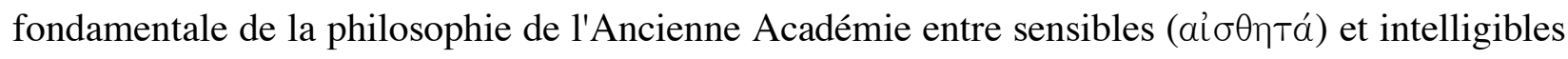
(voךTá). Avant de présenter chacune des huit disciplines mathématiques, ainsi que leurs subdivisions, Géminus-Proclus prononce un certain nombre d'exclusions du champ des mathemata (n'oublions pas le sens premier et général du terme, "connaissances apprises"). Certaines disciplines comme la tactique, l'histoire ou la médecine font un usage occasionnel et

\footnotetext{
15 Comm. in Arstt Phys., B, 2, CAG, IX, 1882, pp. 291-292. V. aussi Géminos, Introduction aux Phénomènes. Ed. et trad. G. Aujac, CUF, Paris, Belles-Lettres, 1975, pp. 111-113.

16 Ibid., pp. XXII-XXIV.

17 Dans son commentaire aux Coniques d'Apollonius (ed. J. L. Heiberg, vol. II, p. 170, 1. 25-26); notons qu'il se réfère au Livre VI de l'ouvrage qui devait donc avoir une certaine extension. Pappus le cite sous le titre $\dot{\eta} \tau \tilde{\omega} \nu$ $\mu \alpha \theta \eta \mu \alpha ́ \tau \omega \nu \tau a ́ \xi_{\iota S}$ (Coll. math., L. VIII, ed. F. Hultsch, p. 1026, 1. 8-9), sans doute une partie introductive, d'où provient notre classification.

18 Pour des traductions françaises, v. Les Commentaires sur le Premier Livre des Eléments d'Euclide. Trad. franç. P. Ver Eecke, 1948. Réimp. Paris, A. Blanchard, pp. 31-36 ou Géminos, op. cit., pp. 114-117.
} 
instrumental de résultats mathématiques. Ce n'est pas pour autant que leurs praticiens doivent être considérés comme des mathématiciens. Il s'agit donc d'un système fermé. Après ces généralités, il reprend chacune des spécialités, dans un ordre un peu différent (Cf. le schéma ci-dessous). Il commence, comme il se doit, par les mathématiques "pures", d'abord la géométrie, puis l'arithmétique (ce qui ne correspond pas à la hiérarchie habituelle). Ces deux sciences possèdent des objets définis (figures et nombres) et c'est également en termes d'objets - et seulement en termes d'objets - que sont distinguées leurs sous-espèces ${ }^{19}$.

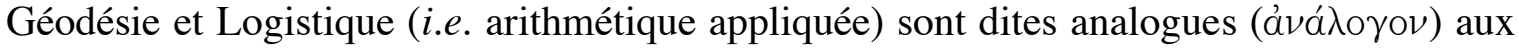
précédentes. On a donc une proportion : ce que la géométrie est à la géodésie, l'arithmétique l'est à la logistique. Il est difficile de parler des objets de ces deux sciences, sauf à dire qu'il s'agit des sensibles, en tant qu'ils participent à la forme et à la quantité. Les rapports internes aux couples ne sont toutefois pas du même ordre. Dans le cas de la géodésie, il s'agit de recourir à des approximations, ou, si l'on préfère, à une géométrisation approchée des sensibles. Dans celui de la logistique, on enfreint les principes mêmes de l'arithmétique, à savoir l'insécabilité de l'unité et l'impossibilité de différencier celles qui composent un nombre ${ }^{20}$.

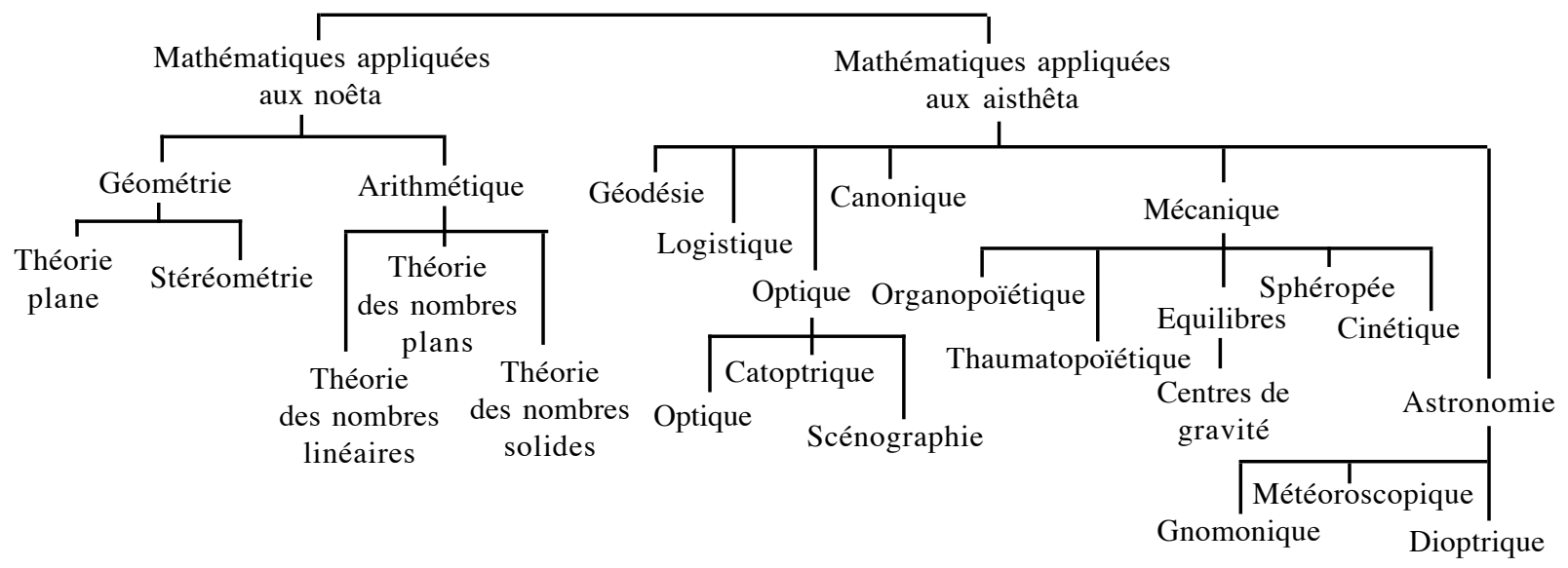

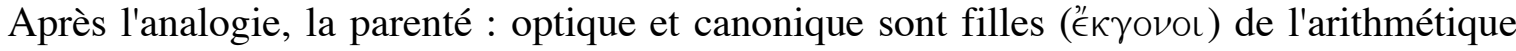
et de la géométrie. La deuxième a un objet clairement identifié par son nom même : la division du canon musical, et ne connaît pas de subdivisions. Quant aux subdivisions de l'optique (optique proprement dite, catoptrique, scénographie), elles ont en commun de rendre compte (par démonstration) de ce qui se montre ( $\tau \grave{\alpha} \phi \alpha \iota \nu o ́ \mu \in \nu \alpha$ ) en ce que cela peut avoir de trompeur.

Suit la mécanique : seule indication différentielle par rapport à ce qui précède en termes

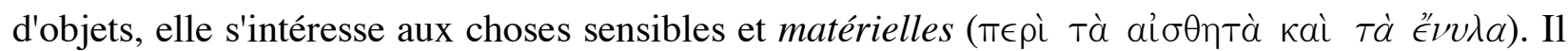
est sans doute difficile de dire que le son, la lumière ou le rayon visuel sont matériels. On doit cependant remarquer qu'il n'y a pas vraiment de définition de la mécanique par son objet : les

\footnotetext{
19 Seule la distinction géométrie plane / stéréométrie a une certaine pertinence par rapport à la pratique des mathématiciens. C'est sans doute ce qui justifie l'inversion de l'ordre « géométrie-arithmétique » puisque les prétendues divisions de l'arithmétique (en théorie des nombres linéaires, plans et solides) s'en inspirent. A son tour, cette inversion justifie l'ordre à l'intérieur des deux couples qui suivent : géodésie-logistique; optique-canonique.

${ }^{20}$ Cf. les Df. VII. 1-2 des Éléments d'Euclide.
} 
deux premières sous-espèces sont désignées à partir du type d'engins qu'elles construisent, des moyens qu'elles utilisent, car la mécanique désigne aussi une partie de la technique des machines. Cela s'applique nettement moins bien à la théorie des équilibres et des centres de gravité ou même à la sphéropée. L'artificialité qui accompagne la notion aristotélicienne de mécanique est déclinée ici un peu différemment : deux des espèces de la mécanique sont présentées comme des

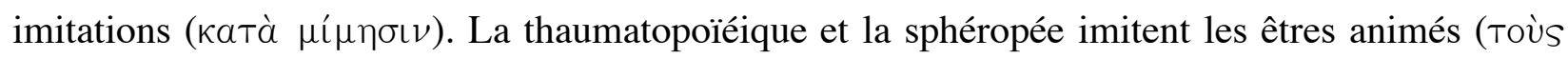

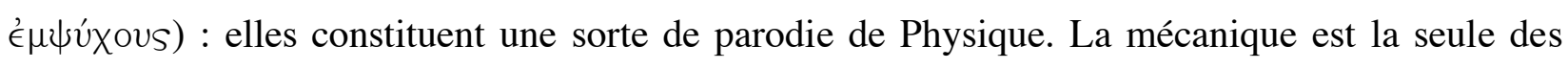
huit sciences pour laquelle Géminus mentionne des noms : Archimède - Ctésibios - Héron ${ }^{21}$, comme si la mécanique se définissait par les mécaniciens ! De fait, le rôle d'Archimède dans la constitution de la mécanique comme science mathématique deviendra une question très débattue à la fin de l'époque hellénistique et au début de la période romaine ${ }^{22}$. A l'inverse, il est bien précisé que l'astronomie est étroitement liée à la physique.

La progression globale de la présentation de Géminus pour les six espèces qui traitent des choses sensibles suit donc une implication physique croissante : d'abord celles pour lesquelles on ne retient que des "caractéristiques" mathématiques : forme et quantité (géodésie; logistique); puis celles pour lesquelles on tient compte de l'organe de perception des "objets" (vue : optique, et ouïe : canonique); enfin celles qui se confrontent à des sensibles dotés de matière, inanimée pour la mécanique, animée en astronomie.

C'est à peu près la même description que nous trouvons dans les extraits d'Anatolius d'Alexandrie, insérés dans l'ultime (et inauthentique) section ( $\left.{ }^{\circ} 138\right)$ des Definitiones attribuées à Héron d'Alexandrie ${ }^{23}$. On possède toute une série de témoignages sur un ou plusieurs Anatolius dont il est difficile de dire s'il s'agit ou non d'une seul et même personnage. Auquel cas, il s'agirait d'un des maîtres de Jamblique, dédicataire d'un de ses traités, lui-même auteur d'un ouvrage d'arithmologie pythagoricienne en 10 livres, chef de l'école aristotélicienne d'Alexandrie et sénateur de cette ville, ultérieur élu évêque de Laodicée ${ }^{24}$. Lesdits extraits, s'ils procèdent bien d'un seul et même auteur, témoignent d'un éclectisme certain. L'extrait 1 fait allusion à la définition aristotélicienne de la mathématique que l'on peut dégager de Métaphysique, E, 1 (mais

\footnotetext{
${ }^{21}$ Sans doute un ajout de Proclus. Il est certainement aussi responsable de la référence au Timée (op. cit., 41, 12).

${ }^{22}$ V. Vitrac, B., « Mécanique et mathématiques à Alexandrie : le cas de Héron ». Oriens-Occidens, à paraître (2004).

${ }^{23}$ Heron Alexandrinus Opera. Ed. J. L. Heiberg. Leipzig, Teubner, vol. IV, 1912, pp. 160, 1. 8-p. 168, 1. 12.

24 V. Goulet, R., DPhA, vol. I, pp. 179-183. On peut ajouter un argument en faveur de l'identité d'auteur pour nos fragments et l'ouvrage d'arithmologie pythagoricienne. L'extrait $\mathrm{N}^{\circ} 6$ est souvent passé sous silence car il paraît un

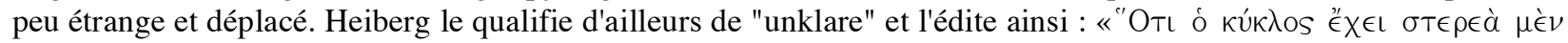

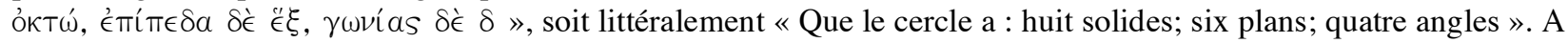
la suite de Th.-H. Martin (qui avait donné une explication de cette note dans ses Recherches sur la vie et les ouvrages

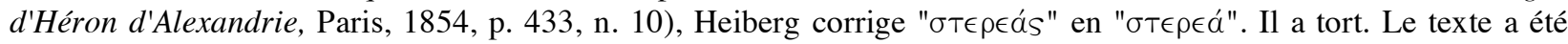

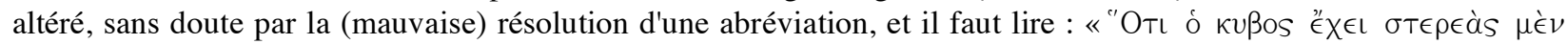

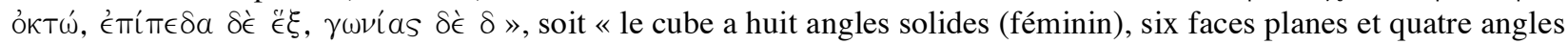
(s. ent. plans par face). Il ne s'agit donc pas de classification d'objets géométriques (comme dans le sauvetage ébouriffant de Martin), mais d'une considération arithmologique banale sur le fait que le cube est une "harmonie" (8, 6, 4). Il manque la mention des 12 arêtes pour faire bonne mesure. On trouve ce genre de remarques chez les auteurs néo-pythagoriciens, tout particulièrement dans les Theologoumena arithmetica attribuée (faussement) à Jamblique, qui contiennent aussi plusieurs passages explicitement attribués à Anatolius.
} 
sans la donner), et conclut, avec Aristote, que la mathématique est une partie de la philosophie.

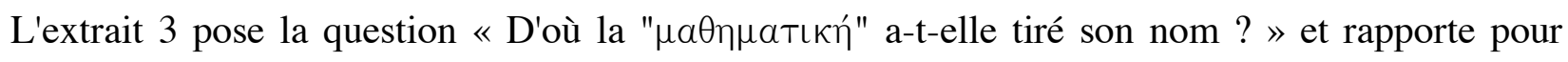

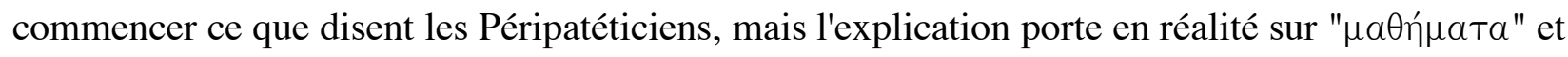
se résume à dire qu'il est impossible d'être autodidacte en mathématiques, contrairement à ce qui se produit en rhétorique, poétique ou musique populaire. C'était devenu un lieu commun fondé sur l'étymologie.

Suit une seconde explication qui n'est autre qu'une généalogie, totalement fictive, du schéma dit de Géminus :

« Mais on dit que Pythagore et ses disciples ont appliqué spécialement ce nom de "mathématique" à la géométrie et à l'arithmétique seules. Car, autrefois, chacune de ces sciences portait un nom différent et n'avait pas de nom commun à l'une et l'autre. Ils les appelèrent donc ainsi parce qu'ils y trouvèrent le caractère scientifique approprié à l'enseignement ... Mais les auteurs récents ont donné une plus grande extension à ce mot, dans la pensée que le mathématicien devait s'occuper, non seulement de la matière incorporelle et idéale, mais encore de celle qui touche à la substance corporelle et sensible.

En effet il doit être adroit dans la théorie du mouvement des astres...; de plus il doit savoir considérer les changements de la vue, et scruter les raisons pour lesquelles les objets ne paraissent pas, à toute distance, ce qu'ils sont, ... Ils pensaient, en outre, qu'un tel homme devait être mécanicien, expert en géodésie et logisticien, et qu'il devait, a fortiori, s'occuper des causes du mélange mélodieux des sons et de leur composition musicale; lesquels sont précisément corporels, ou, du moins, à l'extrême fin de ceux qui s'élèvent au-dessus de la matière sensible $»^{25}$.

Le texte ne prétend pas que les mathématiques "appliquées aux choses sensibles" n'existaient pas autrefois, car il se focalise sur la question du nom. Au départ - c'est-à-dire avant Pythagore ! - chaque spécialité avait son nom propre, sans qu'il y ait de nom commun. Le maitre instaura une communauté entre arithmétique et géométrie - les mathématiques qui portent seulement sur les intelligibles selon Géminus - et ce pour des raisons éducatives. Puis on leur adjoignit les spécialités qui traitent des sensibles. C'est une généalogie des sciences mathématiques, parallèle à la procession néo-pythagoricienne qui s'oppose totalement à la version d'inspiration aristotélicienne que Proclus a conservée dans son célèbre Résumé de l'histoire de la géométrie ${ }^{26}$, même si les deux textes ne se placent pas exactement sur le même plan. Qu'Anatolius ait été intéressé par le problème des relations interdisciplinaires se voit encore dans l'extrait 7. Après avoir rappelé les huit branches principales de la classification (Géminus n'est pas nommé) et prononcé quelques exclusions supplémentaires (extrait 5), il souligne les proximités qui existent entre certaines spécialités (extrait 7) :

\footnotetext{
${ }^{25}$ Op. cit., p. 160, 1. 24-p. 162, 1. 4; 1. 6-14; 1. 20-25. Trad. M. Villermain-Lécolier, légèrement modifiée.

26 Procl., In Eucl. I, ed. Friedlein, p. 64, 1. 7-p. 68, 1. 6. V. Vitrac, B., « Mythes (et réalités ?) dans l'histoire des mathématiques grecques anciennes » dans L'Europe mathématique. Eds C. Goldstein, J. Gray, J. Ritter. Éditions de la Maison des sciences de l'homme. Paris, 1996, pp. 32-51, en particulier pp. 40-46.
} 
«5. ... Ni ce que l'on appelle tactique, ni l'architecture, ni la musique populaire, ni l'étude des phases des étoiles, ni même celle qui est homonyme à la mécanique proprement dite, ne sont, contrairement à ce que pensent certains, des parties de la mathématique, comme nous le démontrerons tout au long de notre exposé avec clarté et méthode.

7. Ce qui se rapproche le plus de l'arithmétique, ce sont la logistique et la canonique... Ce qui se rapproche le plus de la géométrie, ce sont l'optique et la géodésie; et ce qui se rapproche à la fois de l'une et de l'autre au plus haut point, ce sont la mécanique et l'astronomie $»^{27}$.

Pour conclure provisoirement sur le schéma géminien j'ajouterai deux remarques :

1. Il procède d'emblée par une division binaire et les Modernes sont de ce fait tentés de recourir à leurs catégories telles les oppositions «science-technique» ou mathématiques "pures" versus "appliquées", dans lesquelles les premiers termes sont censés être utilisés de manière instrumentale dans la mise en œuvre des seconds. Ce qui s'en rapproche le plus, dans la classification de Géminus, c'est sans soute la distinction entre l'arithmétique et la géométrie d'une part et leurs usages en logistique et géodésie.

Mais, dans le champ des mathématiques tel qu'il apparaît avec la distinction de huit branches principales, mieux vaudrait distinguer trois sortes de disciplines : (i) celles qui s'appliquent seulement aux intelligibles (arithmétique, géométrie); (ii) leurs applications instrumentales (logistique, géodésie); (iii) des spécialités qui, pour dépendantes qu'elles soient des résultats de l'arithmétique et de la géométrie, n'en sont pas moins des spécialités scientifiques autonomes. A ma connaissance, rien d'aussi développé n'a existé dans les autres civilisations anciennes qui ont pourtant développé des mathématiques parfois très sophistiquées. L'astronomie, l'harmonique, l'optique et la mécanique vont bien au-delà d'usages instrumentaux que l'on trouve parfois dans ces cultures, lesquelles ne font pas de distinctions tranchées entre ce qui relève de la nature, par exemple l'étude du Ciel et des astres, seule discipline assez universellement attestée, et ce qui porte sur des artefacts et les pratiques sociales (constructions, arpentage, comptabilité ...). Le regroupement opéré par Géminus est par conséquent un peu forcé. Il faut certainement y reconnaître une influence platonicienne, nous y reviendrons.

2. Il faut être reconnaissant à Proclus de nous avoir conservé ce témoignage détaillé et il faut sans doute en tenir compte dans l'évaluation que l'on porte sur son attitude vis-à-vis des mathématiques. De même qu'il a retenu une version d'inspiration aristotélicienne pour l'histoire de la géométrie plutôt qu'une généalogie fantaisiste « à la Anatolius », il ne s'est pas contenté des sciences du quadrivium comme l'ont fait la plupart des autres médio- et néo-Platoniciens. Il s'est attaché à présenter le schéma le plus large que les Anciens aient élaboré. Sans doute avait-il confiance dans les ressources de son épistémologie pour rendre compte de tous ces développements.

\footnotetext{
27 Op. cit., respectivement p. 164, 1. 13-18 et p. 164, 1. 21-p. 166, 1. 3. Malheureusement nous ne possédons pas l'exposé détaillé promis. Ce témoignage confirme le statut controversé de la mécanique.
} 
En ce qui concerne le quadrivium, Proclus procède en deux temps : 1. Il résume rapidement ce que l'on trouvait déjà dans l'Introduction arithmétique de Nicomaque de Gérase, peut-être en utilisant la "paraphrase" de Jamblique ${ }^{28}$. 2. Sans doute mécontent de ce que le premier avait donné des exemples sensibles (vivant, arbre, tas ...), il entreprend une nouvelle justification du quadrivium, à partir de l'exposé du Timée et en conformité avec la lecture néoplatonicienne du pythagorisme.

Nicomaque invoquait lui aussi ses autorités : Androcyde le pythagoricien, Archytas de Tarente, l'auteur de l'Épinomis (qu'il identifie à tort avec Platon) et enfin Platon lui-même (Livre VII de la République ). De fait, il se place directement dans la lignée d'Archytas dont il prétend citer le début d'un écrit. Il livre ainsi ce qui constitue l'une des versions du fragment DK 47 B1 :

«Certes, ceux qui cultivent les mathématiques me semblent bien discerner; et il n'y a rien d'absurde qu'ils pensent correctement ce qu'il en est au sujet de chaque chose. Car ayant fait preuve d'un bon discernement en ce qui concerne la nature des totalités, ils doivent bien voir ce qu'il en est des choses particulières. Ainsi en ce qui concerne les vitesses des astres, leurs levers et leurs couchers, ils nous ont transmis des évaluations sûres, ainsi qu'au sujet de la

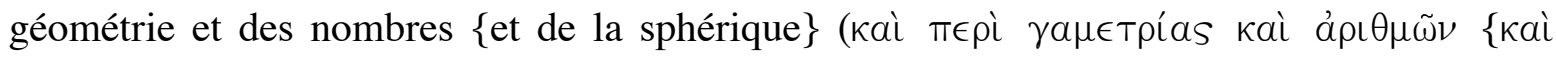

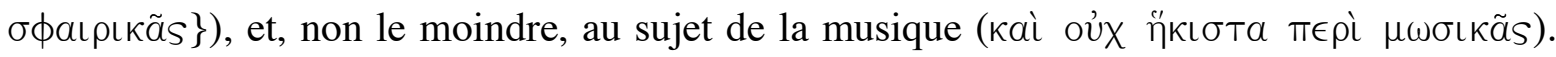
Car ces connaissances paraissent sœurs, puisqu'elles sont tournées vers les deux formes primordiales de ce qui est, qui sont sœurs $»^{29}$.

L'intérêt de la présentation de Nicomaque réside dans le fait qu'il s'agit, non d'une simple énumération, mais d'une articulation raisonnée à partir de trois oppositions polaires. La distinction fondamentale est mérologique : il y a deux types de composition d'un tout à partir de ses parties, soit divisé, de manière juxtaposée, comme en un tas, soit de manière unifiée et en état de cohésion. Aristote indique une division comparable de la quantité entre ce qui est, en puissance, divisible en choses non continues et ce qui l'est en choses continues ${ }^{30}$. Cette distinction, bien établie, gouverne aussi la distinction des Livres géométriques (I-VI+XI-XIII) et arithmétiques (VII-IX) des Éléments d'Euclide. Les sous-distinctions internes à ces deux modes d'êtres : « en soi / en relation »; « en repos / en mouvement » ne sont pas homogènes. Elles tirent vraisemblablement leur origine de discussions platoniciennes. On peut résumer l'ensemble sous la forme d'un tableau :

\footnotetext{
${ }^{28}$ Cf. Procl., In Eucl. I, ed. Friedlein, p. 35, 1. 21 - p. 36, 1. 8, Nicom., Intr. arith., ed. Hoche, p. 4, 1. 13-p. 6, 1. 7, Jambl., In Nicom. Ar. intr., ed. Pistelli, p. 7, 1. 2-p. 9, 1. 1. Les notions utilisées par ces trois auteurs, les désignations des quatre sciences sont les mêmes. Mais certains termes, notamment ceux qui désignent les objets de la géométrie et de la sphérique sont un peu différents. Proclus est plus proche de Jamblique que de Nicomaque.

${ }^{29}$ Diels \& Kranz, 1985, t. I, pp. 431, 1. 36 - 432, 1. 8. Diels a confronté Nicom., Intr. arith., L. I, III, 4, éd. Hoche, pp. 6, 1. $17-7,1.2$ et Porphyre, Commentaires aux Harmoniques de Ptolémée, éd. Düring, p. 56. 1. 2-10.

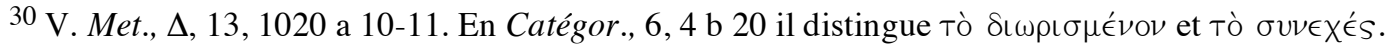




\begin{tabular}{|c|c|c|}
\hline [ÊTRES] & $\begin{array}{l}\text { DISCRETS } \\
\delta ı \eta \rho \eta \mu \epsilon ́ v a\end{array}$ & 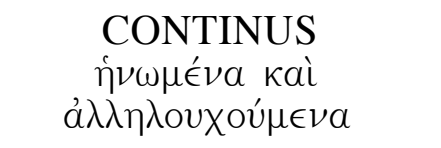 \\
\hline $\begin{array}{l}\text { INDETERMINEE } \\
a ̊ a t \pi \in \rho \alpha\end{array}$ & $\pi \lambda \tilde{\eta} \theta \mathrm{os}$ & $\mu \epsilon^{\prime} \gamma \in \theta$ os \\
\hline $\begin{array}{l}\text { DETERMINEE } \\
\pi \epsilon \pi \epsilon \rho \alpha \sigma \mu \epsilon ́ v \alpha\end{array}$ & 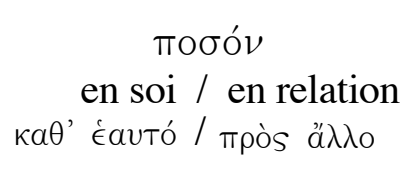 & 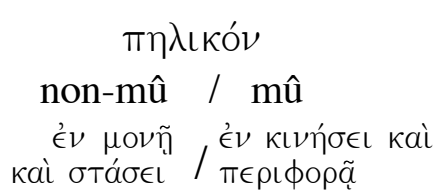 \\
\hline Sciences correspondantes & Arithmétique / Musique & Géométrie / Sphérique \\
\hline
\end{tabular}

Que ce schéma ait été désigné comme pythagoricien ne doit pas surprendre, compte tenu de son environnement textuel. Le début de l'Introduction (Ch. I et II, §§ 1-3) se place sous le patronage de Pythagore, même si les doctrines présentées sont platoniciennes, et la présentation du quadrivium se referme avec la citation du fragment d'Archytas. Nicomaque a sans doute perçu que son schéma suggérait un certain éclatement du système des sciences mathématiques, ce qu'il s'efforce d'atténuer de deux manières. Il proclame l'unité de l'ensemble des quatre disciplines en citant un passage de l'Épinomis ${ }^{31}$, où il croit lire une telle affirmation; il souligne que toutes ces sciences sont comme des échelles et des ponts, qui permettent de faire passer notre pensée des sensibles aux intelligibles. Rien là de très original, ni de très convaincant.

Qui plus est, la fidélité de Nicomaque vis-à-vis de ses sources n'est pas vraiment exemplaire. Puisqu'il cite le Livre VII de la République, il aurait dû présenter cinq sciences et non pas quatre, ainsi que le fait, par exemple, Théon de Smyrne dans son Expositio. Sans doute étaitil difficile de justifier la distinction « géométrie / stéréométrie » selon un critère ontologique. De même, pour les quatre sciences retenues, il n'a pas conservé l'ordre platonicien. En rapprochant arithmétique et musique d'une part, géométrie et sphérique d'autre part, il renforce la prégnance de son critère mérologique et il produit aussi une articulation plus proche de la description aristotélicienne. Plus gênante encore est sa manière de transmettre le fragment d'Archytas. D'abord il n'a pas conservé le dialecte dorien maintenu dans la version de Porphyre. Diels retient donc celle-ci, mais en la complétant à l'aide de Nicomaque ! Notons :

- que là où Porphyre dit « au sujet des nombres » (ảpı $\theta \mu \tilde{\omega} \nu)$, Nicomaque cite l'arithmétique.

- que la mention de la sphérique n'existe pas chez Porphyre;

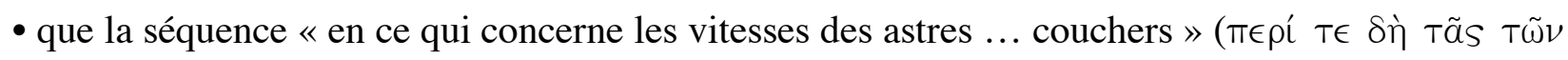

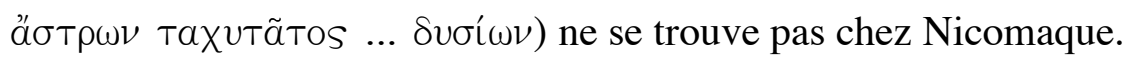

- Enfin, que le commentaire ontologique «puisqu'elles sont tournées vers les deux formes primordiales de ce qui est, qui sont sœurs » est absent du texte de Porphyre !

Outre les substitutions, qui pourraient paraître bénignes, Nicomaque a clairement manipulé le texte : il a supprimé la séquence sur les astres et lui a substitué la sphérique, insérée

31991 e - 992 b, désigné comme treizième livre des Lois que certains, dit Nicomaque, appellent le philosophe. 
en avant-dernière position. D'où une complète homogénéisation des désignations des quatre disciplines dans le fragment cité, avec celles qu'il a lui-même données juste avant. Ajoutons que "sphérique", dans le contexte du IVe siècle avant notre ère, voire plus tôt si Archytas parlait de certains de ses prédécesseurs, fait totalement anachronique. Surtout Nicomaque change le sens de la dernière phrase, peut-être même de l'ensemble du texte. En rapprochant sphérique et musique, ce sont elles - et peut-être seulement elles - qui sont qualifiées de sciences sœurs. Ce qui devient un évident clin d'œil à l'affirmation similaire de Platon (pour "astronomie" et "harmonique"), au Livre VII de la République (530 d8), placée sous le patronage des Pythagoriciens explicitement nommés !

Si on garde le texte de Porphyre, ce pourrait être l'ensemble des sciences mathématiques qui forment une famille. On peut même aller plus loin. Si Platon a raison dans l'identification des sciences sœurs, il faut comprendre qu'il est fait ici mention de deux spécialités seulement : d'une part une étude du cosmos (vitesses, levers et couchers des astres, mesure de la Terre - un sens possible de "géométrie"32 - et les nombres que cela implique : périodes, éloignements relatifs, mesures), d'autre part, la musique, objet de l'exposé qui suit ${ }^{33}$ ! Qu'il faille y lire une classification quadripartite des sciences ne va donc plus de soi. Cette lecture n'a d'autre justification que l'interprétation confirmative de Nicomaque. L'enracinement "ontologique" du propos d'Archytas, qui découle du dernier membre de phrase, est lui aussi, et malgré Diels, le résultat d'une des manipulations que l'Auteur de l'Introduction a fait subir au texte.

\section{IV}

A la charnière des premiers siècles avant et après notre ère - l'époque de Géminus - les

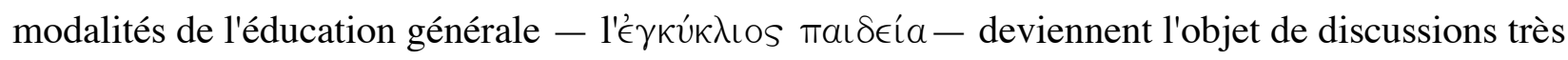
nourries ${ }^{34}$. Avec Nicomaque (début du $\mathrm{II}^{\mathrm{e}}$ siècle), le quadrivium est constitué en tant que tel. Il est disponible pour composer, avec les trois spécialités du trivium (grammaire, rhétorique et dialectique), le cycle des sept arts libéraux. Celui-ci, en tant que modèle d'éducation proposé « aux hommes libres » est formellement attesté au IVe siècle de notre ère dans le néoplatonisme latin. Dans ce contexte, on peut, à la suite de $\mathrm{M}^{\mathrm{me}}$ Hadot, vouloir faire la différence entre deux ordres de questions, selon qu'on envisage le quadrivium comme une réalité socio-éducative ou comme un schéma idéal. En tant que réalité éducative, il a sans doute existé dans les écoles du Moyen, puis du Néo-platonisme, mais non pas comme forme généralisée de l'éducation. Ainsi, à l'époque impériale, alors qu'il y a institution de l'enseignement des disciplines du trivium, rien de tel n'existe pour celles du quadrivium. Par conséquent, celui-ci n'existait probablement pas en tant que réalité éducative générale à l'époque hellénistique, même si les modalités réelles de l'enseignement scientifique de cette période nous sont bien mal connues. Une chose est sûre : le

\footnotetext{
32 Cf. Aristophane, Nuées, v. 200-216.

33 L'étroitesse du sujet traité comparativement à la cosmologie, si l'on suit l'interprétation que je donne ici, justifie le

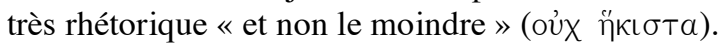

34 V. Hadot, I., Arts libéraux et Philosophie dans la pensée antique. Paris, Études Augustiniennes, 1984.
} 
quadrivium, en tant que description d'un ensemble de savoirs mathématiques, n'est pas une invention de l'Antiquité impériale ou tardive car on le trouve, à plusieurs reprises chez Platon, notamment dans le programme éducatif du Livre VII de la République ${ }^{35}$. Ceci pouvait conforter l'hypothèse de Nicomaque, Jamblique et Proclus quant à l'origine pythagoricienne du système à quatre sciences.

Si les différentes descriptions anciennes des savoirs mathématiques qui nous sont parvenues proviennent d'un contexte philosophique, les questions qu'elles soulèvent vont bien audelà, d'abord, parce qu'aussi bien chez Platon que chez Aristote, la réflexion sur le sujet s'appuie sur la réalité des pratiques des mathématiciens de leurs temps, ensuite, parce que cette mise en ordre raisonnée des savoirs s'inscrit dans une entreprise anthropologique beaucoup plus vaste, qui concerne l'ensemble des activités humaines. Déjà au $\mathrm{V}^{\mathrm{e}}$ siècle avant notre ère, les historiens, les médecins et les auteurs du théâtre athénien partagent cette idée que l'espèce humaine en tant que telle se caractérise par sa maîtrise progressive de ce qu'on appellera ensuite "science" et "technique". Ce cadre très général est, par exemple, totalement explicite dans l'Épinomis ${ }^{36}$. D'où un intérêt pour les énumérations des TéXval sous forme de listes, leur origine ( emprunt / don divin), leur inscription dans une série d'oppositions réglant les différentes

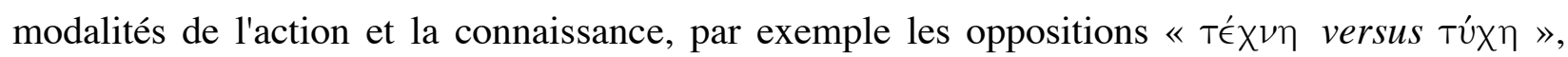

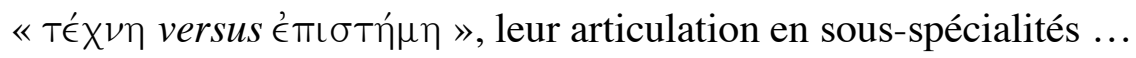

Ainsi une scholie liminaire à la Tekhnê (grammatikê) de Denys le Thrace affirme :

«Et il faut savoir, à propos de toute tekhnê, que l'on doit examiner huit items. Et ce sont les

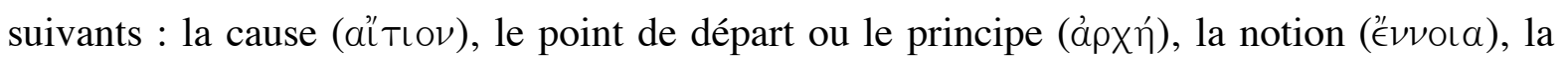

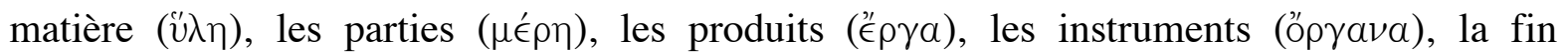

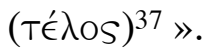

Le point de départ de cette réflexion réside sans doute en partie dans le fait que les termes

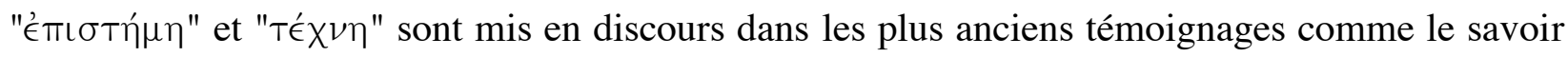
ou l'expertise d'un agent. Cela est d'ailleurs encore vrai dans certains passages de Platon et dans les définitions d'Aristote et, sans doute, cela ne sera jamais perdu de vue. Il n'y a d'ailleurs pas de raison à cela. Mais avec le développement considérable et la diversification des pratiques savantes et techniques, on a sans doute perçu la nécessité, au-delà de l'unité subjective du polymathe, de pratiquer certains découpages et regroupements, bref d'objectiver des disciplines. Et, pour pallier le risque d'un éventuel éclatement, de spécialisation outrancière d'ailleurs dénoncée par quelques-uns, il a fallu, dans le même temps, exhiber des relations mutuelles entre

\footnotetext{
35 Mais, selon $\mathrm{M}^{\text {me }}$ Hadot, ce programme a été abandonné par l'Académie dès l'époque d'Arcésilas et de Carnéade (vers $260^{\mathrm{a}}$ ). Il est réintroduit seulement dans la nouvelle Académie, à l'époque de Cicéron.

${ }^{36}$ En 974 d 8 - 976 b $5+990$ c 5 - 992 a 6, l'Auteur distingue :

- les arts visant à la satisfaction des nécessités (agriculture, art de bâtir, artisanat, chasse; divination);

- ceux qui visent l'agrément, essentiellement d'ordre "mimétique" (dessin, peinture, poésie, musique, rhétorique...);

- les arts du secours (art de la guerre, du pilote, de l'avocat; la médecine);

- enfin les sciences : arithmétique, géométrie, stéréométrie, astronomie et musique.

37 V. Scholia in Dionysii thracis artem grammaticam. A. Hilgard (ed). Partis primæ Volumen tertium, Leipzig, Teubner, 1901, pp. 106-124, en particulier p. 113, 1. 11-12.
} 


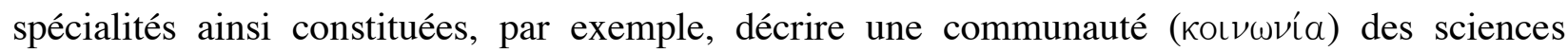
mathématiques.

Ceci peut se faire de différentes manières. On peut se référer à l'histoire, mais le développement empirique des différentes recherches risque d'être quelque peu chaotique. On pourrait chercher les fondations du côté des facultés humaines. Il semble que c'est ce que feront les auteurs de l'Encyclopédie, au XVIII ${ }^{e}$ siècle ${ }^{38}$. Dans le cas des disciplines mathématiques telles qu'elles sont décrites par les philosophes grecs du IV $\mathrm{V}^{\mathrm{e}}$., les sciences se distinguent et s'articulent d'abord en fonction de la nature de l'objet ou des objets sur le(s)quel(s) elles portent. C'est l'un des usages habituels qu'en font les dialogues socratiques, jusqu'au Gorgias, lorsqu'ils cherchent à déconsidérer ce qu'ils présentent comme les généralités des sophistes et des rhéteurs. L'argument commence généralement avec des TéXvar productrices. Les spécialités mathématiques sont là pour montrer que cela vaut aussi pour les disciplines intellectuelles qui ne fabriquent pas leur objet. Ce critère ontologique, que l'on retrouve dans les classifications, fonctionne plutôt bien pour l'arithmétique, la géométrie, l'astronomie, l'harmonie, autrement dit les sciences du quadrivium; cela fonctionne également avec l'optique ainsi qu'avec les sous-espèces de ces différentes sciences. Cela marche nettement moins bien - c'est un euphémisme - avec la mécanique.

Quant aux relations mutuelles, les classifications elles-mêmes sont un peu décevantes. Pour l'essentiel elles se contentent de métaphores familiales : «sciences sœurs », dans le

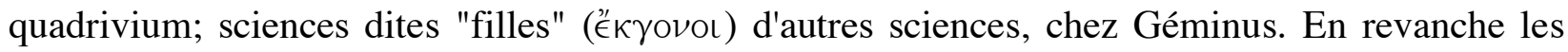
programmes éducatifs renforceront la communauté de ces disciplines à l'aide de critères "externes", par exemple en leur assignant une méthode et un projet commun - la connaissance dianoétique $^{39}$ et une fonction propédeutique à la dialectique chez Platon - ou en circonscrivant ceux qui doivent y avoir accès, par exemple l'élite des gardiens-philosophes dans la Cité idéale du Livre VII de la République. Le Philèbe, moins extrémiste, procède un peu différemment. Après avoir montré que le champ des téXvaı s'organise selon deux pôles (55 d5-56 c7), l'un qui représente la part de conjecture, d'exercice et d'expérience accumulée, l'autre qui correspond à la quantification, Socrate distingue ensuite (56 c8-57 e2), au sein même des arts mathématiques, deux parties selon qu'ils sont pratiqués par le vulgaire $(\dot{\eta} \tau \tilde{\omega} \nu$ по $\lambda \lambda \tilde{\omega} \nu)$ ou le philosophe $(\dot{\eta} \tau \tilde{\omega} \nu$

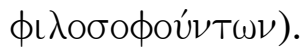

Isocrate unifie lui aussi les mathématiques - il mentionne les calculs, la géométrie et l'astronomie - par leur finalité et leurs destinataires, quand bien même ses choix sont très différents de ceux de Platon. Il ne s'agit pas de souligner le caractère intelligible des objets mathématiques, mais les vertus pédagogiques qu'elles possèdent, au même titre que l'éristique, en tant qu'exercices intellectuels. L'opposition «jeunes / hommes faits » sous-tend son analyse : ce

\footnotetext{
38 V. d'Alembert, Discours préliminaire de l'Encyclopédie. Paris, Vrin, en particulier la description du système figuré des connaissances humaines, pp. 180-181, commandée par la distinction : Mémoire-Raison-Imagination, et l'explication qu'en donne d'Alembert pp. 163-174. Pour les mathématiques (pp. 169-171) cela lui permet tout de même d'intégrer, pratiquement sans variations, certaines portions de la classification dite de Géminus !

${ }^{39}$ Les plus optimistes y verront une préfiguration d'une classification par les "facultés".
} 
sont des activités à réserver à la jeunesse qu'il serait ridicule de poursuivre trop longtemps. L'homme fait doit s'occuper des affaires privées et publiques ${ }^{40}$. Xénophon soutient une position analogue, encore plus étroite, tout en se réclamant de l'enseignement de Socrate ${ }^{41}$ ! Pour nos trois auteurs athéniens, au début du IV siècle, les considérations sur les sciences mathématiques interviennent dans un contexte pédagogique, et donc politique. C'est pourquoi, malgré leurs nuances, les programmes de la République et des Lois mêlent inextricablement d'une part, des considérations épistémologiques (idéalités des objets mathématiques - et tout particulièrement idéalité de l'unité et du nombre) et philosophiques (opposition de ce qui est toujours et de ce qui toujours devient, ou de la perception et de l'intellection) et, d'autre part, des remarques d'ordre sociologique (distinction de l'élite philosophique par opposition à la foule, des philosophes par rapport aux praticiens) ou prescriptives (pratiquer les sciences pour elles-mêmes, voire pour une fin plus élevée encore, et non selon une visée utilitaire). Entre Xénophon et Platon, il s'agit aussi de savoir qui est le véritable héritier de Socrate.

\section{V}

Si tel est le cadre général dans lequel apparaissent différentes énumérations des sciences mathématiques, voire des classifications sous forme de programme raisonné, reste à déterminer les conditions historiques précises dans lesquelles elles ont été élaborées et quelle a été l'attitude des mathématiciens. Ce qui suppose l'examen d'un problème préalable : cela a-t-il un sens de spéculer sur la différence entre "mathématiciens" et "philosophes", de supposer qu'il existait déjà, au IVe siècle avant notre ère, voire avant, une division du travail intellectuel et une spécialisation si nettes qu'elles permettent l'utilisation de telles étiquettes sans qu'il s'agisse d'un grossier anachronisme. Les historiens des sciences ne se sont pas toujours embarrassés de cette question. Ainsi, au début du $\mathrm{XX}^{\mathrm{e}}$ siècle, l'idée de «philosophe-géomètre » en Grèce ancienne - sans doute fallait-il comprendre "pythagoricien" ou "platonicien" - était assez largement partagée. Le fait qu'il y ait eu, dans la culture grecque, une interaction forte, continue et réciproque entre mathématique et philosophie, depuis les Milésiens et les Éléates jusqu'aux Néo-platoniciens n'a été remis en cause qu'assez récemment, à la suite des travaux d'historiens anglo-saxons, d'abord Otto Neugebauer en ce qui concerne l'astronomie, puis Wilbur Knorr pour les mathématiques, au sens étroit du terme. Cela dit, admettre, à la suite de ces deux auteurs, qu'il a existé une spécialisation scientifique autonome indépendante des questionnements philosophiques n'empêche pas de penser qu'il s'agit d'un processus. Leurs analyses se fondent, pour l'essentiel, sur l'analyse des traités techniques conservés des époques hellénistique et impériale. Reste donc la possibilité de s'interroger sur les débuts de cette spécialisation. Qu'en était-il, en particulier, au $\mathrm{Ve}^{\mathrm{e}}$ siècle avant notre ère ? Clairement il s'agit d'un cas particulier du problème de la

\footnotetext{
40 V. Busiris, § 23; Antidosis, §§ 261-265; Panathénä̈que, §§ 26-30.

41 V. Mémorables, IV, VII, §§ 2-9. Platon fait d'ailleurs dire la même chose à Calliclès (Gorgias, 484 c5-d2) à propos de la philosophie et le trait deviendra lui aussi un lieu commun des débats éducatifs.
} 
« disciplinarisation » des savoirs. Marie-Laurence Desclos a repris récemment cette thématique. Elle souligne qu'à partir du milieu du $\mathrm{V}^{\mathrm{e}}$ siècle, certaines Té Xval, notamment la médecine et la rhétorique, ont revendiqué leur singularité en tant que savoir, que cela n'allait pas de soi et ne valait pas pour l'iбторía. On remarquera cependant que l'histoire ne sera jamais véritablement

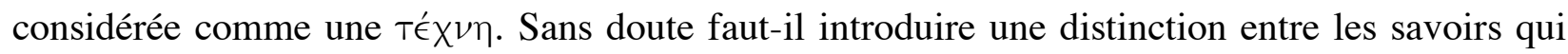
peuvent prétendre jouer un rôle prépondérant dans l'éducation : poésie, histoire, rhétorique et philosophie d'un côté et, de l'autre, les TéXval, toujours associées à la notion de savoir spécialisé. C'est ce qui permet de regrouper, sous une même étiquette, des choses aussi variées que les métiers artisanaux ou artistiques, la médecine, la géométrie, l'astronomie ${ }^{42}$... En fait, selon M.-L. Desclos, la division du champ du savoir en territoires distincts doit beaucoup à Platon ${ }^{43}$.

Qu'en est-il des spécialités mathématiques ? Si l'on suit les auteurs de l'Antiquité tardive, le schéma global du début des mathématiques est à peu près le suivant : les spécialités mathématiques ont été inventées par les Barbares pour satisfaire certaines nécessités; des Sagesvoyageurs les ont rapportées en Hellade (les candidats les plus fréquents sont Thalès et Pythagore, mais il y a aussi Enopide de Chio, Démocrite, Eudoxe ...). Surtout Pythagore en a fait un schéma éducatif : le quadrivium. La « disciplinarisation » est donc antérieure à Platon. Peu d'historiens acceptent aujourd'hui ces mythes d'origine. Au demeurant, ils ne répondent pas à notre question car, quelle que soit la contribution que l'on reconnaisse aux anciens Pythagoriciens, il sera difficile d'y voir des scientifiques totalement détachés des spéculations philosophiques. La même remarque vaudra, non seulement pour les premiers Milésiens (Thalès, Anaximandre), mais aussi pour des personnages comme Démocrite, Archytas et Eudoxe - dont la chronologie est à peu près parallèle à celle de Socrate, Platon et Aristote ! - et que la tradition présente à la fois comme mathématiciens et philosophes ${ }^{44}$.

Mais nous possédons deux ou trois portraits dans lesquels il est tentant de reconnaître de "purs" mathématiciens et ce, dans des dialogues de Platon, qui plus est dans des passages où les sciences du quadrivium sont explicitement énumérées. Il s'agit du sophiste Hippias d'Élis et des géomètres Théodore de Cyrène et Théétète d'Athènes. Le cas de ce dernier, contemporain et ami de Platon - la tradition rapporte même qu'ils ont l'un et l'autre étudié la géométrie avec Théodore - est un peu plus délicat si l'on admet qu'il a appartenu à l'Académie, davantage encore s'il a participé à des discussions du genre de celles que le Théétète et le Sophiste mettent en scène. En revanche, Hippias, d'après le portrait qu'en brosse Platon, est un sophiste certes polymathe, mais pas philosophe. De même Théodore, présenté comme un disciple de Protagoras,

\footnotetext{
42 Ce que fait Théétète (146 c8-d2) en associant les sciences mathématiques, l'art des cordonniers et toutes les

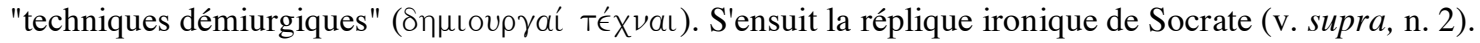

43 Desclos, M.-L., Aux marges des dialogues de Platon, Grenoble, Éditions Jérôme Millon, 2003, p. 6, 8, 63-64 et tout particulièrement la section pp. 154-163.

44 Même si le cas de « Démocrite mathématicien » est un peu plus délicat à analyser. V. Vitrac, B., «L'interprétation mathématique du dilemme du cône (DK 68 B 155). Démocrite était-il mathématicien ? » dans Les anciens savants. Études sur les philosophies préplatoniciennes réunies par P.-M. Morel et J.-F. Pradeau. Les Cahiers Philosophiques de Strasbourg, Tome 12, Automne 2001, pp. 89-129.
} 
est peu porté, à cause de son âge dit-il, aux discussions philosophiques ${ }^{45}$. Aucun des deux n'est pythagoricien. Pourtant Platon décrit leurs activités mathématiques à l'aide du schéma quadripartite :

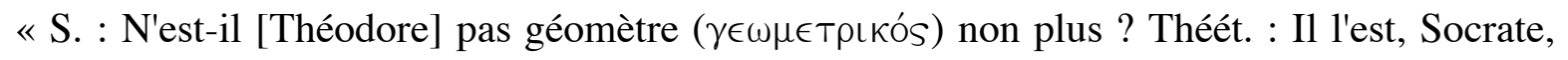

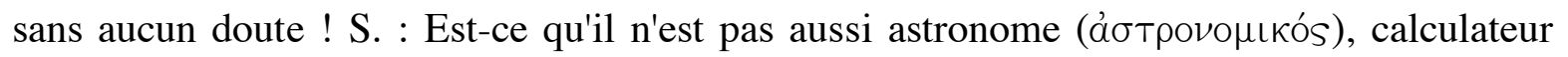

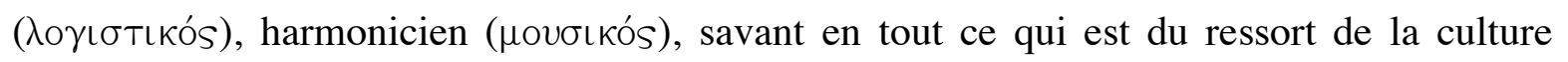

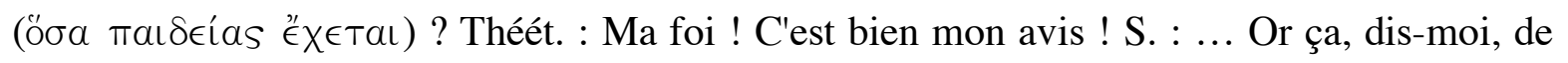
Théodore tu apprends ( $\mu \alpha \nu \theta a ́ v \in \mathrm{IS})$, je suppose, certaines choses de géométrie $(\gamma \in \omega \mu \in T \rho i ́ \alpha) ? . .$. Et aussi certaines de celles qui se rapportent à l'astronomie, l'harmonie

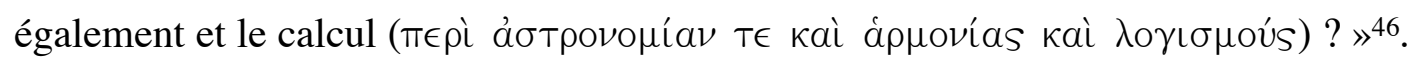

Réalité historique ou construction platonicienne ? Si l'on penche pour la première solution, l'hypothèse que certains intellectuels non philosophes, appartenant à (ou proche de) la première Sophistique, aient pu jouer un rôle dans la mise en discipline des sciences mathématiques », en liaison avec le renouveau éducatif auquel on associe ce mouvement, devient plausible.

Relisons également le témoignage du Protagoras, à propos d'Hippias :

«Les autres sophistes en effet font du mal à la jeunesse; car, alors que celle-ci s'est éloignée des disciplines spéciales ( $T E ́ \chi \chi V S$ ), ils l'y ramènent contre son gré et la précipitent dans l'étude de spécialités ( $\epsilon$ is TéXvas) en leur enseignant le calcul, l'astronomie, la géométrie, la musique ( ce disant, il tourna les yeux du côté d'Hippias $»^{47}$.

Il existe d'autres indices : les tentatives de quadrature du cercle attribuées par Aristote à Antiphon et Bryson (quand bien même il les juge a-géométriques); le fait qu'Isocrate puisse mentionner l'astronomie, le calcul et la géométrie comme composantes recommandables pour l'éducation dans son Busiris, vers 390, donc probablement avant la fondation de l'Académie. Citons enfin la réfutation des géomètres, qu'Aristote attribue à Protagoras, et qui témoigne que ce mouvement ne parlait pas d'une seule voix sur ces questions ${ }^{48}$. C'est d'ailleurs ce que Platon fait dire au sophiste d'Abdère dans le dialogue qui porte son nom. Toutefois, s'il paraît permis de parler de mathématiciens non philosophes, il ne s'agit pas de spécialistes, à l'exception possible du seul Théodore. Leur polymathie et la variété de leurs préoccupations intellectuelles est précisément l'un des reproches que Platon leur adresse.

Si l'on admet cette hypothèse, on comprendra que Platon se soit intéressé à cet embryonnaire système des sciences, qu'il ait voulu le reprendre à son compte, tout en exigeant une profonde réforme des disciplines concernées et en leur proposant une finalité différente.

\footnotetext{
45 V. Theaet., 146 b1-4.

46 Theaet., resp. 145 a5-8 et 145 c7-d3 : Trad. L. Robin. Paris, Gallimard, 1950, vol. II, pp. 87-88. Pour Hippias, les choses sont un peu moins claires (v. infra, n. 51), mais Platon utilise le schéma quadripartite à son propos dans le Protagoras.

47 V. Protagoras, 318 d9-e4. Trad. L. Robin. Paris, Gallimard, 1950, vol. I, p. 85.

48 Met., B, 2, 998 a3-4.
} 
Réforme que l'Antiquité tardive attribue à Pythagore ! En rattachant ce schéma éducatif aux Pythagoriciens, on a du même coup éliminé les Sophistes du paysage. Ce genre d'hypothèses est difficile à éprouver. Cela dit, on peut au moins montrer que le quadrivium n'était pas la seule façon de présenter les sciences mathématiques aux Ve-IVe siècles. Il ne s'agissait donc pas d'un système canonisé depuis des décennies.

Il existe un ensemble de textes, y compris platoniciens, qui mentionnent seulement trois sciences mathématiques : calcul ou arithmétique, géométrie ou métrétique et astronomie. La musique en est soit totalement absente, soit rattachée aux études littéraires ${ }^{49}$. Deux lignes d'interprétation sont possibles : (i) ou bien il s'agit de listes sans prétention à former un système, et elles pourraient se rattacher au système quadripartite par synecdoque; (ii) ou bien il faut imaginer qu'il a existé une sorte de "trivium" mathématique.

A l'appui de cette seconde hypothèse, on remarquera que la mention des Lois (817 e5-9) se rapporte à un programme éducatif, cette fois pour les hommes libres d'une nouvelle colonie et non pas pour les gardiens-philosophes d'une Cité idéale, et qu'il est donc difficile de l'expliquer par l'incomplétude fortuite de l'énumération. On peut ajouter à cela qu'Eudème de Rhodes a écrit les histoires de ces trois disciplines mathématiques seulement, sans doute parce que ce sont celles qu'Aristote considérait comme "hégémoniques". Ainsi le seul fragment de l'histoire de l'arithmétique identifié (le $\mathrm{N}^{\circ} 142$ ) attribue aux Pythagoriciens la découverte des rapports numériques associés aux intervalles de quarte, de quinte et d'octave. Autrement dit, il s'agit d'harmonique qui, pour les Péripatéticiens, est subordonnée à l'arithmétique. A certains égards, on peut même dire que ce "trivium" mathématique se retrouve dans l'Antiquité impériale et tardive, par exemple chez Ptolémée et Proclus lui-même, quand ces auteurs nous expliquent qu'il y a trois "objets" mathématiques : le nombre, la grandeur et le mouvement. La différence de traitement que subit la musique s'explique sans doute d'abord par l'ambiguïté du terme, et par le fait que musique et gymnastique composaient l'étape élémentaire de l'éducation traditionnelle.

Clairement cet écart a été perçu par les Anciens eux-mêmes et a donné lieu à une élaboration "historique" intéressante. Le point de départ en est sans doute le fait que les témoignages du Phèdre de Platon et du Busiris d'Isocrate se situaient en milieu égyptien. D'où l'affirmation, rapportée par Diogène Laërce ( $\$ 11$ de son introduction) et déjà par Diodore de Sicile, que l'arithmétique, la géométrie et l'astronomie ont été inventées par les Égyptiens. Diodore précise même que les jeunes Égyptiens ne pratiquent ni la gymnastique, ni la musique. Autrement dit, ce ne sont pas Grecs ${ }^{50}$. Rien d'étonnant donc à ce que, dans leurs Vies de Pythagore, Porphyre, puis Jamblique, expliquent que la géométrie a été découverte en Égypte Jamblique fait allusion à Hérodote —, l'arithmétique l'a été par les Phéniciens et la science du

\footnotetext{
49 Attestent la première possibilité : Euthydème, 290c, Phèdre, 274 c5-d2; Gorgias, 450c-451 c5; Lois, 817 e5-9; Isocrate, Busiris, § 23; Xénophon, Mémorables, L. IV, Ch. 7, 2-9; Aristoxène de Tarente, Harmoniques, Livre II, préface. Quant à la seconde possibilité, elle est illustrée dans deux autres témoignages platoniciens concernant Hippias d'Élis (Hipp. mai, 285 b5-d5; Hipp. min., 366c5-368 d6) et dans le catalogue des œuvres de Démocrite établi par Thrasylle et transmis par Diogène Laërce (Vies, L. IX, §§ 47-48).

${ }^{50}$ Cf. Biblioth. hist., L. I, § LXIX, 5; § LXXXI, 1-5 et 7.
} 
Ciel par les Chaldéens selon Porphyre, par les Égyptiens ou les Chaldéens pour Jamblique.

Cette équitable répartition explique les multiples voyages de Pythagore et justifie qu'on le considère comme l'inventeur du quadrivium. Clairement, en effet, s'il a rapporté ces trois sciences en Grèce et les a perfectionnées, il en a ajouté une quatrième. Pour ces auteurs, la musique mathématique (= la théorie des intervalles) est indubitablement son invention. Prudents, Aristote et son disciple Eudème l'attribuent aux Pythagoriciens ${ }^{51}$. C'est une belle histoire puisqu'aux trois sciences mathématiques d'origine barbare et empirique - l'arithmétique est née du commerce; la géométrie de l'arpentage; l'astronomie de la navigation et du calcul calendérique - Pythagore a ajouté la moins utilitaire, la plus esthétique et sans doute la plus divine des sciences du système quadripartite. La découverte des rapports numériques associés aux intervalles musicaux fondamentaux peut bien entendu servir à fabriquer des flûtes justes mais, selon les Pythagoriciens, le domaine privilégié dans lequel se manifestait cette structure mathématique qui s'exprime dans la théorie des médiétés était la cosmologie ${ }^{52}$. Belle histoire donc, mais très improbable!

Pour revenir à l'époque classique, nous pouvons discerner chez certains auteurs des $\mathrm{Ve}^{\mathrm{e}}$ $\mathrm{IV}^{\mathrm{e}}$ siècles encore une autre description des savoirs, conçue selon trois niveaux que je qualifierai de cosmologiques : ce qui est sous la terre, ce qui est dans le ciel, ce qui est sur la terre (la géométrie). Cette description est celle d'Aristophane, dans les Nuées (jouée en 423 et reprise en 418-416). On la retrouve chez Platon lui-même, dans le Théétète, quand il prétend citer Pindare et qu'il décrit la Sıávoı du philosophe qui étend partout «son vol, à ce qui est sous la terre, à la surface de laquelle elle pratique la géométrie, comme, sur la voûte qui domine le ciel, elle pratique l'astronomie ... ${ }^{53}$. Moins systématique, l'auteur de l'Ancienne Médecine - un traité qui appartient à une couche ancienne du corpus hippocratique - critique vertement ceux qui ont recours aux hypothèses pour discourir sur les choses invisibles par exemple « au sujet des météores ou sous la terre » (I. 3). Une telle description n'est pas vraiment en accord avec celles que proposent les schémas en trois ou quatre sciences. A tout le moins il faut supposer que l'on a là des descriptions divergentes.

Tout aussi significatif que ce que l'on trouve chez Platon est ce qui en est absent. Nous possédons des témoignages sur les débuts de l'optique, mis en rapport avec la scénographie dans le théâtre attique à l'époque d'Eschyle (première moitié du Ve $\mathrm{s}$.) ${ }^{54}$. Même si on récuse ces informations, les Météorologiques d'Aristote montrent que certaines études d'astronomie et de

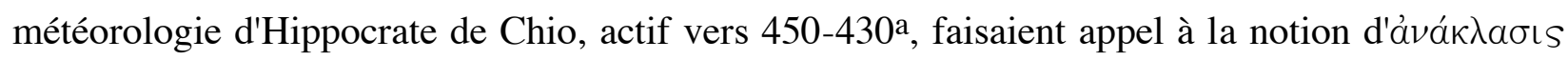
du rayon visuel. Le Stagirite rapporte notamment plusieurs explications portant sur la (sic )

\footnotetext{
51 V. Arstt, Met., A, 5, 985 b30 - 986 a10; Eudème, Frgt Wehrli Nº142.

52 Signalons toutefois que l'origine barbare de l'harmonie n'est pas exclue par tous les auteurs. Ainsi Jamblique, sans contredire Diodore, affirme que Pythagore apprit la médiété parfaite chez les Babyloniens.

53 Theaet., 173 e4-174 a1. Trad. L. Robin. Paris, Gallimard, 1950, vol. II, p. 132.

54 Voir Vitruve, De architectura, Livre VII, préface, qui mentionne : Agatharcus, instruit par le dramaturge athénien Eschyle, Démocrite (cf. Catalogue des écrits in Diogène Laërce, Vies, IX. 4) et Anaxagore.
} 
comète et sur la nature de la Voie lactée. Parmi celles-ci, il discute sérieusement celle d'Hippocrate qui prétendait rendre compte de la rareté des apparitions de la comète et de ce que l'on ne pouvait pas observer sa chevelure à chaque fois ${ }^{55}$. Son explication, en termes d'élévation au-dessus de l'horizon (ce qui supposait vraisemblablement des trajectoires circulaires et un cosmos sphérique), était de nature géométrique et optique : l'apparition de la chevelure dépendait de la "rupture" de nos rayons visuels, à cause de l'humidité ambiante.

Notons au passage que ce sont les mêmes principes qu'Aristote mobilisera dans son explication de l'arc-en-ciel (mais cette fois Hippocrate n'est pas cité), dans le chapitre le plus mathématisé de toute son œuvre conservée (Météorol., III. 5). En outre, la tradition rapporte que Philippe d'Oponte, disciple et "secrétaire" de Platon, avait composé, parmi d'autres écrits mathématiques, des Optiques et des Catoptriques, chacun en deux Livres. Alexandre d'Aphrodise, quand il commente l'analyse aristotélicienne de l'arc-en-ciel, atteste que Philippe utilisait le même genre d'explications qu'Aristote et Hippocrate. Apulée attribue pour sa part la théorie du rayon visuel issu de l'œil à $\operatorname{Archytas}^{56}$. Même si ce témoignage est plus incertain, il paraît difficile de contester que des questions "optiques" existaient déjà à l'époque de Platon et qu'elles intéressaient les géomètres. Celui-ci n'y fait cependant jamais référence en tant que spécialité mathématique. A l'inverse, il évoque ce genre de phénomènes comme exemples d'erreurs de la vision, illustrant la faiblesse de la perception comme instrument de connaissance. Quant à l'invention de la mécanique mathématique, elle est rapportée au même Archytas de Tarente $^{57}$ et, sans être obligé de croire ce qu'en dit Plutarque ${ }^{58}$, on doit constater que cette discipline n'apparaît pas non plus dans les dialogues. Sans doute cela s'explique-t-il par l'implication matérielle de la mécanique. Ce survol des témoignages confirme le développement considérable des recherches mathématiques à l'époque de Platon et d'Aristote ${ }^{59}$, ainsi que des tentatives, variées, de décrire et d'organiser ces savoirs. Ces tentatives sont sans doute à l'origine de nos deux classifications transmises par les auteurs postérieurs. Celles-ci ont donc bien, au moins en un sens large, un rapport avec l'activité des mathématiciens. Dans le cas de la classification dite de Géminus, le lien est confirmé a posteriori par la comparaison avec les traités conservés des époques hellénistique, puis impériale et tardive, et ce, de deux manières.

- Certaines spécialités correspondent, au moins par synecdoque à des titres d'ouvrages : Optiques; Mécaniques; Harmoniques, attestés dès les débuts de l'époque hellénistique; Arithmétiques, Logistiques, Géodésie utilisés dans l'Antiquité tardive.

\footnotetext{
55 Météor., I, 6, 342 b 29sq; en I, 8, 345 b 9 il mentionne une explication similaire pour la Voie lactée; Alexandre d'Aphrodise précise qu'elle était également due à Hippocrate.

56 Pour la distinguer des théories épicurienne et platonicienne. V. DK 47 A 25.

57 Diogène Laërce, Vies, VIII, § 83.

58 Vie de Marcellus, 14. 9-11; Propos de tables, VIII, question 2, 718 E 7-F4. Plutarque et Diogène Laërce se réfèrent très certainement à une source commune, car l'un et l'autre estiment que ces premiers travaux concernaient le problème de la duplication du cube. Ce pourrait être Ératosthène de Cyrène qui, lui aussi, proposa une solution au même problème et en fit l'histoire, depuis Hippocrate de Chio (et même depuis Minos !) jusqu'à lui-même.

${ }^{59}$ Cf. Euclide, Les Éléments. Trad. et comm. par B. Vitrac. Collection Bibliothèque d'histoire des sciences. Paris, P. U. F. Volume 2 : Livres V à IX, 1994, p. 24, n. 64.
} 
- La quasi-totalité des écrits mathématiques conservés se laisse ranger dans l'une des cases de la classification de Géminus, parfois plusieurs, dans le cas des ouvrages de taille importante, par exemple les Éléments d'Euclide (géométrie : L. I-VI + XI-XIII; arithmétique : L. VII-IX) ou la Collection mathématique de Pappus (géométrie : L. III-IV-V, VII; astronomie-optique : L. VI; mécanique : L. VIII). C'est alors le découpage interne en Livres qui respecte à peu près celui des spécialités distinguées par la classification. Il y a donc homologie entre ce schéma et la manière dont les mathématiciens, non pas poursuivent leurs recherches, mais rédigent leurs ouvrages. Les institutions savantes d'Alexandrie (la Bibliothèque ?) ont peut-être canonisé ce mode de rangement.

\section{VI}

Peut-on aller un peu plus loin et identifier ceux à qui l'on doit nos deux classifications ? Les historiens des mathématiques grecques adoptent souvent la position suivante :

- Le quadrivium pythagoricien est... pythagoricien, comme en témoigne le fragment DK 47 B 1 d'Archytas. Platon le leur reprend, en particulier au Livre VII de la République (Cf. la mention des sciences sœurs) et y ajoute la stéréométrie.

- La classification de Géminus représenterait un état plus développé et plus tardif. L'arithmétique et la géométrie auraient été dédoublées pour distinguer mathématiques "pures" et "appliquées"; l'optique et la mécanique, sciences nouvelles de l'époque, auraient été ajoutées par souci de complétude.

Pour certains historiens, la distinction entre nos deux classifications reflèterait les changements sociaux et politiques qui se sont opérés à la fin de l'époque classique. Le quadrivium correspondrait aux mathématiques développées dans les cercles savants du monde des Cités, tandis que la classification de Géminus représenterait une extension analogue à l'élargissement de l'horizon géographique consécutif aux conquêtes d'Alexandre. La mise en place des monarchies hellénistiques et le développement du mécénat royal, tel celui de la dynastie des Lagides à Alexandrie, soucieux d'applications pratiques, auraient bénéficié aux mathématiques qui traitent des sensibles, tout particulièrement à la mécanique. Le géomètre se serait détaché du philosophe pour se rapprocher de l'ingénieur60. D'autres, et ce n'est pas contradictoire, ont vu, dans l'élaboration de cette classification élargie, l'influence du Lycée et $\mathrm{d}^{\prime}$ Aristote ${ }^{61}$, particulièrement attentif aux possibilités d'appliquer les mathématiques à certains aspects du monde physique. Je n'adhère plus à cette description classique. Je crois :

- que la fixation du quadrivium est en grande partie due à Platon. Il ne me paraît pas possible de dire s'il a entièrement inventé ce schéma - qu'il aurait rétroprojeté sur des auteurs antérieurs ou s'il l'a emprunté à d'autres. Dans cette seconde hypothèse, il me semble que le cercle de Théodore de Cyrène est un candidat au moins aussi bon que les Pythagoriciens.

\footnotetext{
60 Kline, M., Mathematical Thought from Ancient to Modern Times. Oxford University Press, 1972. Edition Paperback en 3 volumes, 1990, vol. I, pp. 104-105.

${ }^{61}$ C'est ce que j'ai moi-même suggéré op. cit., pp. 22-24.
} 
- que les grandes lignes de la classification de Géminus étaient déjà fixées à l'époque de l'Ancienne Académie. Ce proto-schéma était connu de - et critiqué par - Aristote. Le Stagirite propose d'articuler autrement le même ensemble de disciplines. Reprenons chacun de ces points.

La qualification traditionnelle du quadrivium a peu d'autorité. Nous avons vu ce qu'il en est des élucubrations concernant Pythagore dans les textes postérieurs à l'époque hellénistique. L'examen du fragment $1 \mathrm{~d}$ 'Archytas n'est guère plus concluant quand on mesure les distorsions qu'un Nicomaque lui fait subir, sans doute en toute bonne foi, convaincu par avance de ce qu'il fallait y lire. En ce qui concerne les mathématiques pythagoriciennes, il faut nous contenter de ce que disent les sources les plus anciennes, en l'occurrence Platon, Aristote et Eudème de Rhodes : les Pythagoriciens ont développé une étude des principes premiers des mathématiques, le nombre et les figures simples, laquelle comportait un aspect "logique" en ce sens qu'il y avait une hiérarchie et sans doute une génération desdits principes.

Surtout ils les ont mis en œuvre dans deux disciplines (qualifiées de sœurs) : l'astronomie et l'harmonie. D'après le témoignage fondamental de République, VII, il ne s'agissait plus d'astronomie parapegmatique ou nautique, selon la désignation que l'on veut bien donner aux formes les plus anciennes de la recherche astronomique grecque (étude des levers et couchers d'astres en conjonction avec des prédictions météorologiques), mais d'une étude des astres attentifs à leurs mouvements, leurs périodes et leurs éloignements mutuels. Le problème de la Grande Année et la structure mathématique de l'âme du monde qui régit ces mouvements dans le Timée est peut-être un écho de ces recherches. De même l'harmonique n'est pas seulement la recherche empirique sur l'accord des instruments, mais une étude de la consonance des intervalles. Platon trouve cette approche pythagoricienne insuffisante.

Cette description est en accord avec ce que rapporte Aristote et j'ai suggéré que l'on pouvait lire le fragment $1 \mathrm{~d}$ 'Archytas dans cette optique, sans qu'on ait besoin de préjuger de son authenticité. Ce témoignage est décisif pour l'attribution du quadrivium à une (très ancienne) école pythagoricienne parce qu'on suppose qu'Archytas, en mentionnant ceux « qui étudient les mathématiques », se réfère à ses prédécesseurs et maîtres. Or, même si l'on admet la lecture en termes de quadrivium, rien n'interdit de penser qu'il parle simplement de mathématiciens antérieurs. Il faut ici rappeler que Platon utilise ce schéma pour décrire les activités mathématiques d'Hippias et de Théodore, l'un et l'autre certes antérieurs à Archytas, mais pas pythagoriciens !

Quant à Archytas lui-même, si l'on accorde du crédit au fait qu'il a consacré une partie de ses travaux à l'optique et à la mécanique géométriques, il serait un peu paradoxal d'en faire le promoteur d'un système en quatre sciences seulement. A tout prendre, c'est un meilleur candidat pour la mise en place d'un système intégrant les disciplines principales de la classification dite de Géminus, à condition toutefois qu'il se soit soucié de classifier les sciences mathématiques.

On pourrait rapporter une première élaboration du quadrivium aux Sophistes qui se sont intéressés aux mathématiques et l'on pense immédiatement au témoignage de Platon sur Hippias dans le Protagoras, d'autant que la discussion porte sur l'enseignement sophistique. C'est possible 
et la dimension érudite d'autres investigations du sophiste d'Élis est assez consonante avec une recherche taxinomique portant sur les disciplines. Ainsi, dans son célèbre Résumé de la Géométrie $^{62}$, Proclus cite Hippias d'Élis comme autorité historique sur cette science. Or, taxinomie et récit historique allaient souvent de pair dans les considérations sur les Té Xval. Ma réticence vient des deux autres témoignages platoniciens concernant Hippias qui décrivent ses activités mathématiques plutôt comme un trivium, l'étude des harmonies étant associée aux études littéraires et poétiques ${ }^{63}$. Il se pourrait donc que le témoignage du Protagoras contienne une part d'exagération platonicienne, d'ailleurs justifiée par le contexte : l'opposition entre l'unique enseignement de Protagoras et la polymathie d'Hippias.

Si l'on se souvient de ce que nous avons vu au sujet de la géométrie et de l'astronomie, plusieurs fois associées selon un modèle que j'ai rapproché des niveaux cosmologiques, il semble assez probable que le quadrivium se soit constitué comme une sorte de synthèse entre différentes options de présentation des spécialités mathématiques, options respectivement associées à certains sophistes, aux Pythagoriciens, aux savants "ioniens" ... Cette synthèse pourrait avoir été faite dans le cercle de Théodore de Cyrène - auquel Platon est censé avoir appartenu. Son témoignage sur ce géomètre ${ }^{64}$, là aussi dans un contexte éducatif, est sans ambiguïtés : Théodore est un maître es quadrivium. On notera en particulier la dualité entre le maître et le disciple

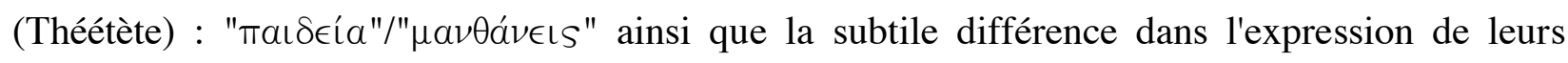
centres d'intérêt commun. Pour Théétète, on énumère les noms habituels des quatre disciplines :

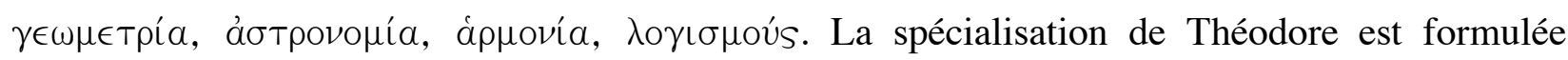

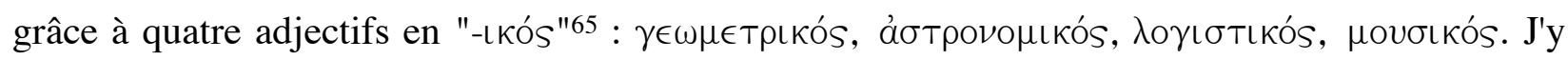
vois un indice, parmi d'autres, de ce que la fixation du quadrivium est, pour l'essentiel, due à Platon.

Les adjectifs en "-ıкós" sont très nombreux chez lui en particulier, mais pas seulement, dans le Sophiste et le Politique. On y trouve de nombreux néologismes et même un certain nombre d'hapax. Selon les linguistes ${ }^{66}$, une des fonctions des termes de cette famille est précisément de marquer l'appartenance à un groupe dans une classification. C'est particulièrement

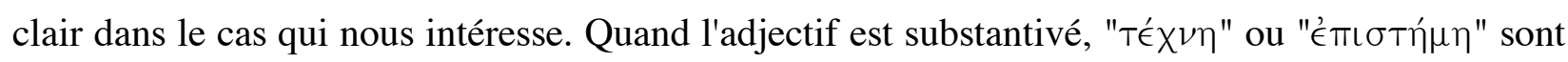
souvent sous-entendus, mais aussi " $\gamma \nu \tilde{\omega} \sigma \mathrm{s} s "$ comme on le voit en Resp. VII, 527 b3-8 pour la «

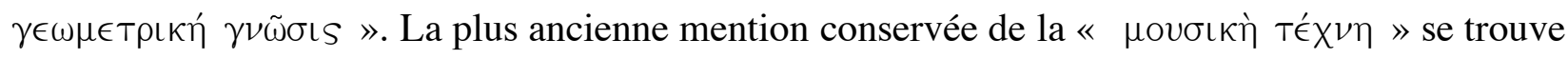
dans le discours d'Éryximaque (Banquet, 187 b2).

\footnotetext{
62 In Eucl., I, ed. Friedlein, p. 65, 1. 14.

63 Références supra, n. 49.

${ }^{64} \mathrm{~V}$. la citation supra, référence in n. 46.

65 En 143 d on souligne que la discipline dans laquelle il excelle au plus haut point est la géométrie. Même chose dans les Mémorables de Xénophon (L. IV, II, § 10). Dans le Politique (257 a7-8), il a le titre d'expert absolu au sujet

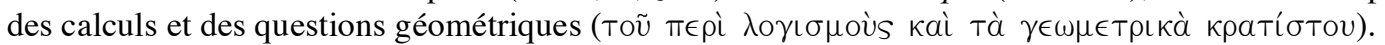

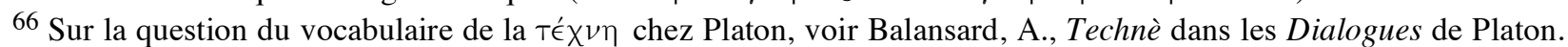
L'empreinte de la sophistique. International Plato Studies, volume 14. Sankt Augustin, Academia Verlag, 2001, en particulier pp. 31-45.
} 
La mise en place du quadrivium pourrait donc faire partie du découpage du champ des savoirs auquel il s'est abondamment livré si l'on suit M.-L. Desclos. Pour les sciences mathématiques, plusieurs innovations linguistiques, certaines distinctions conceptuelles ont été apparemment introduites par Platon. Certaines s'imposeront, d'autres non. Par exemple, s'il n'a évidemment pas inventé l'étude des nombres, on lui doit certaines manières de les désigner et de les concevoir philosophiquement. Il a probablement inventé le terme" arithmétique" et peut-être celui de "logistique" (à moins qu'il ne l'ait repris à Archytas). Il a même essayé de préciser quelle pouvait être la communauté et la différence que l'on pouvait établir entre ces deux études (théoriques) du nombre. Établir ce point réclamerait une argumentation complexe ${ }^{67}$, mais cette contribution partielle à la classification des sciences me paraît incontestable, quand bien même on refuserait de lui accorder davantage. Sa suggestion a eu un succès partiel : le vocable "arithmétique" s'est imposé immédiatement; en revanche "logistique" n'a pas eu cette fortune. Aristote ne l'utilise pas et s'en tient au traditionnel "logismoi" (calculs). Quand "logistique" réapparaît, dans la classification de Géminus, il ne s'agit plus d'une discipline théorique, mais de l'arithmétique appliquée aux sensibles.

Le programme éducatif du Livre VII de la République contient plusieurs traits frappants.

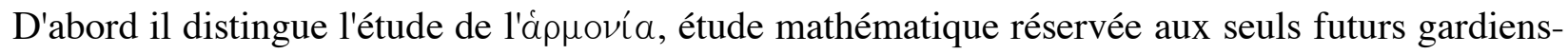

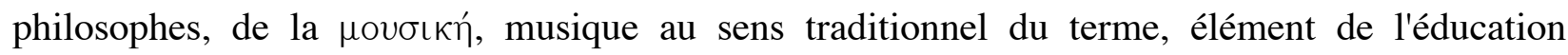
commune à tous. Il introduit une distinction supplémentaire (en $528 \mathrm{ab}$ ) entre la géométrie plane

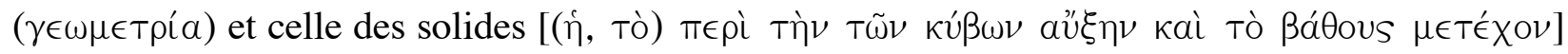
à laquelle il accorde le troisième rang. Le scénario même suivi par Platon d'une erreur de Socrate, qui aurait voulu brûler les étapes, permet de souligner qu'il ne s'agit pas d'une simple énumération, mais d'une classification articulée autour d'une opposition polaire fondamentale,

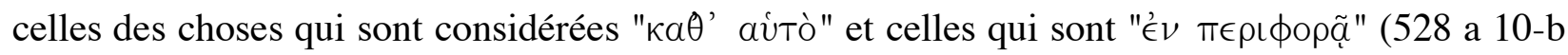
1), d'où une articulation : «arithmétique + géométrie (+ stéréométrie) versus astronomie + harmonique ». Elle ne coïncide donc pas avec celle que présentera Nicomaque. Platon, pour sa part, insiste sur la parenté entre astronomie et harmonie comme sciences qui impliquent la prise en compte du mouvement (í фopá, 530d).

Bien entendu, comme l'a remarqué M.-L. Desclos pour d'autres savoirs ${ }^{68}$, l'auteur de la République ne se prive pas de critiquer la manière dont ceux-ci étaient cultivés par ses prédécesseurs. Outre la critique explicite de l'astronomie et de l'harmonique pythagoriciennes, on

\footnotetext{
${ }^{67}$ Le problème tient à ce que les Dialogues n'adoptent pas une position constante sur cette question. De plus nous manquons cruellement de sources parallèles. L'analyse de Resp. VII, $522 \mathrm{c}-525 \mathrm{~d}$, montre qu'il existe, dans l'étude

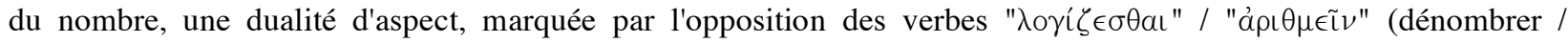
calculer), ce qui n'empêche pas Platon de dire qu'il s'agit d'un seul et même savoir (théorique). Celui-ci est parfois appelé "logistique", parfois "arithmétique", soit dans des dialogues distincts, soit dans le même dialogue comme Resp. VII et le Philèbe, dans lesquels les deux termes sont substituables. Le seul passage qui les oppose est Gorgias, 450 c7-451 c10. V. Caveing, M., La figure et le nombre. Recherches sur les premières mathématiques des Grecs. Lille, Presses Universitaires du Septentrion, 1997, pp. 159-172; Klein, J., Greek Mathematical Thought and the Origin of Algebra (trad. angl. par E. Brann de « Die Griechische Logistik und die Enstehung der Algebra »). Cambridge (Mass.), M. I. T. Press, 1968. Réimpr. New York, Dover Publications, 1992, pp. 17-25.

${ }^{68}$ Elle mentionne d'ailleurs le cas des mathématiques, op. cit., p. 181.
} 
mentionnera celle du langage, risible, de la géométrie (527 a), la mégalomanie de certains de ses spécialistes (des sophistes ? 528 c) et le rejet, plusieurs fois répété dans le Livre VII, mais aussi dans les Lois et le Philèbe, de la pratique intéressée, voire mercantile, de ces disciplines. D'où, selon Platon, la nécessité de légiférer sur leur enseignement, ce qui ne devait pas correspondre à grand chose dans la réalité scolaire grecque. Nous avons vu aussi que le choix platonicien passait probablement par des exclusions, celle de l'optique et de la mécanique. Il y avait d'ailleurs de bonnes raisons à cela :

- Les mathématiciens dont il décrit les compétences, Hippias d'Elis et Théodore de Cyrène appartiennent à une époque plus ancienne et ne semblent pas s'être intéressés à ces questions.

- La mécanique, comme nous l'avons vu encore chez Géminus-Proclus, n'a pas vraiment d'objet parfaitement défini alors que l'identification d'un savoir par son objet est une thématique importante pour le maître de l'Académie.

- Ni la mécanique, ni l'optique ne semblaient en mesure de servir la finalité que Platon impose aux études mathématiques pour la formation des futurs magistrats-philosophes.

Quant à la бтатькń (Charmide, 166 b1-3; Philèbe, 55 e2), ancêtre potentiel de la scientia

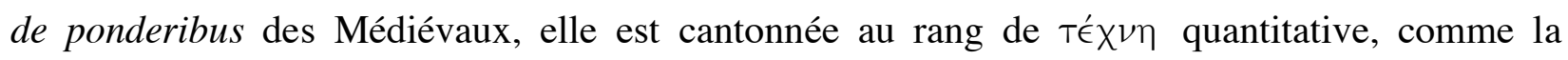

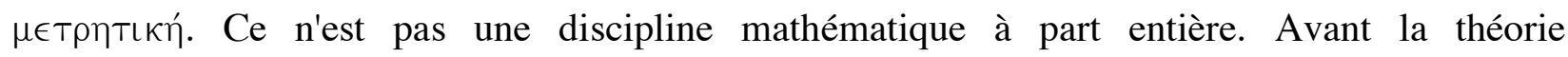
archimédienne des centres de gravité, l'intérêt théorique de la бтатıкń était sans doute difficile à percevoir.

Qu'il y ait un lien évident entre quadrivium et programme philosophique élitiste se voit aussi par contraste avec le schéma éducatif proposé aux citoyens des Lois. : dans ce cas, pas de quadrivium, mais un système plus limité, avec trois études traditionnelles, calcul, mesure, astronomie. Il s'agit également de limiter les prétentions des mathématiciens et ce, d'ailleurs, que Platon ait repris le quadrivium à ses prédécesseurs ou qu'il l'ait articulé lui-même. Légiférer, exclure, (re-)nommer, sont de bonnes opérations pour ce faire. C'est lisible dans la dernière opération de (re-)nomination que Socrate propose à la fin de son exposé du programme. Alors

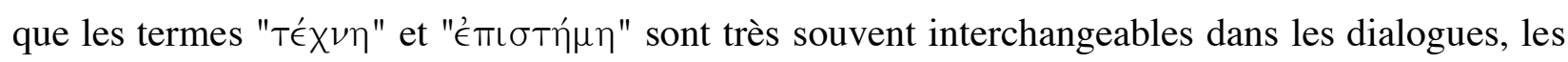
disciplines mathématiques - la géométrie faisant office de porte-drapeau - sont introduites en 533 b7 dans la continuité de tous les autres TéXval, en ce qu'elles ne saisissent pas le réel, ce qui est réservé à la seule dialectique. Platon reconnaît qu'il a cédé à l'usage en les appelant "sciences"

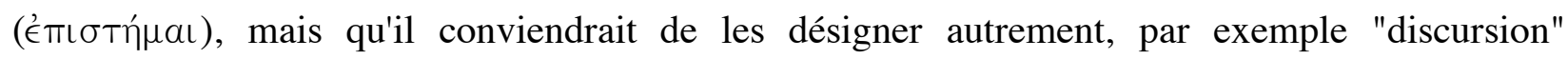

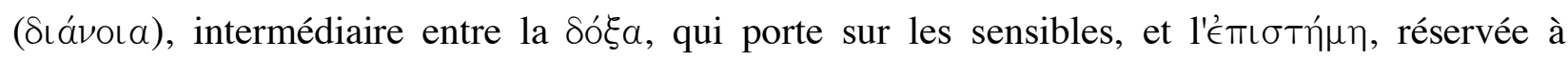
l'appréhension anhypothétique des formes intelligibles, c'est-à-dire à la seule dialectique. Même s'il n'insiste pas - la question du nom, remarque-t-il, n'est pas essentielle - la place des sciences mathématiques, sur la Ligne des savoirs (VI, 509 d-511 e), sera seconde et leur finalité essentiellement propédeutique. Il est amusant de constater que le critère qui justifie ce rang, la position d'hypothèses admises et non justifiées, est l'une des caractérisations qu'Aristote donnera de la démonstration scientifique ! 


\section{VII}

Que dire de l'origine du schéma de Géminus ? Si l'on accepte la description qui précède, il n'y a pas de raison de croire qu'il s'agit d'un développement historique du quadrivium. Précisons d'abord qu'il ne faut pas espérer trouver, chez les auteurs du IVe siècle avant notre ère, un schéma aussi complet que celui rapporté par Proclus, avec ses spécialités elles-mêmes divisées en sousdisciplines. Ainsi les divisions de la mécanique sont très clairement à mettre en relation avec les travaux des savants de l'époque hellénistique, Ctésibios, Archimède, Philon de Byzance, puis Héron d'Alexandrie. Mais on peut imaginer qu'il a existé, dès l'époque de l'Académie, un proto-

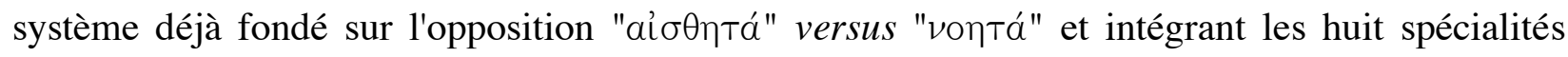
principales, voire quelques subdivisions. Il y a plusieurs indices de cela :

- Nous avons vu que les débuts des recherches en optique et en mécanique remontent certainement à la charnière des $\mathrm{V}^{\mathrm{e}}-\mathrm{IV}^{\mathrm{e}}$ siècles, peut-être avant. Ces deux spécialités sont désignées, sans ambiguïtés, comme des disciplines mathématiques par Aristote.

- Celui-ci mentionne également la géodésie, en contraste avec la géométrie, qui plus est dans un

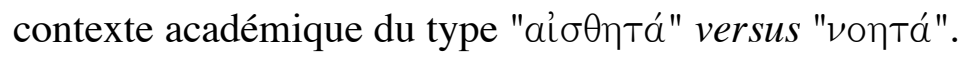

- Il ne cite pas la logistique, mais cela peut s'expliquer par sa proximité chronologique avec la tentative platonicienne de distinguer une logistique théorique de l'arithmétique. A cette exception près, il connaît donc sept des huit disciplines principales de Géminus !

- Des traités intitulés Optiques, Catoptriques, sont attribués à Philippe d'Oponte.

- Il est vraisemblable que des études gnomoniques avaient déjà été entreprises à cette époque puisque Hipparque critique les déterminations de latitude (grâce aux rapports gnomoniques) qu'Eudoxe de Cnide avait proposées et qu'Aratos aurait reprises sans les corriger.

- La distinction géométrie plane-stéréométrie a été introduite par Platon et reprise par Aristote.

Il est donc indubitable que la "matière" d'un tel proto-système existait à l'époque d'Aristote et d'Eudoxe. Nous ne pouvons toutefois pas affirmer qu'il y ait eu une élaboration sous forme de classification ou de programme articulé, une sorte d'alternative au quadrivium platonicien. La chose n'est cependant pas invraisemblable car le principe qui aurait commandé un

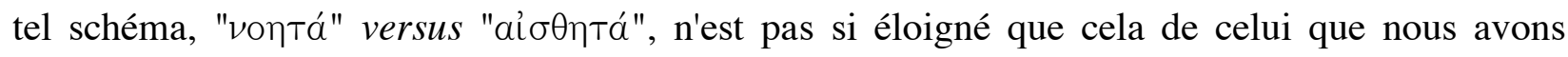

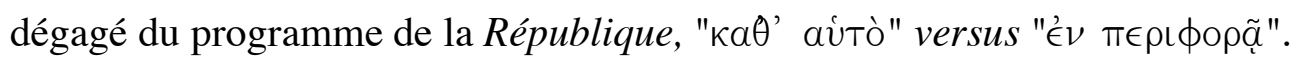

Certains historiens considèrent même qu'un principe de ce genre se retrouve déjà dans le tableau des TéXvaı du Philèbe ${ }^{69}$, en particulier avec la distinction de deux arithmétiques, l'une pour le vulgaire, manipulant des choses nombrées, l'autre pour les savants utilisant les nombres monadiques. Socrate précise bien qu'il s'agit de deux connaissances qui portent le même nom, mais qu'elles doivent néanmoins être distinguées à cause de leur différence dans l'échelle de l'exactitude et de la pureté (57 b5-c3). Il se peut que des auteurs ultérieurs aient précisément voulu mettre un terme à cette homonymie, en introduisant l'opposition « arithmétique versus logistique », telle que nous l'avons vue chez Géminus.

6955 d1-57 a4 + 57 b5-e5. V. Caveing, M., op. cit., p. 165 et 169-172. 
Quoi qu'il en soit, certains mathématiciens, contemporains de l'élaboration de la République, ont pu ne pas apprécier l'exclusion de certains domaines d'étude impliqués dans le sensible, comme l'optique, la gnomonique ou la mécanique, non plus que l'orientation fondamentalement anti-empirique que Platon assigne à l'astronomie et à l'harmonique. Faute de témoignages, on doit se contenter d'évoquer ici les cercles d'Archytas ou d'Eudoxe ${ }^{70} \ldots$ On sait que Ménechme, lui-même disciple d'Eudoxe, n'évitait pas les discussions avec les membres de l'Académie où, dit-on, il avait étudié. Proclus rapporte un débat qui l'avait opposé à Speusippe et Amphinomos, à propos du statut théorématique de la géométrie ${ }^{71}$. Ce milieu était propice à de tels débats sur l'organisation du savoir mathématique; l'école qu'Eudoxe avait fondée à Cyzique en était un autre.

L'attitude d'Aristote conduit à penser qu'un tel proto-schéma a bel et bien été esquissé. Dans la quatrième aporie du Livre B de la Métaphysique, il pose la question suivante :

« Doit-on reconnaître seulement les substances sensibles ou d'autres en dehors de celles-ci, et si oui, y en a-t-il un seul genre, ou plusieurs, comme le pensent ceux qui posent, outre les Formes, les choses intermédiaires, objets des sciences mathématiques, entre intelligibles et objets de perception ? $\gg^{72}$.

Le contexte de la discussion présuppose des distinctions proches de celles de la classification de Géminus. En outre, dans sa théorie de la démonstration, le Stagirite propose une autre articulation, pour le même ensemble de disciplines, qui me paraît plus sophistiquée que ladite classification.

L'explicitation de la quatrième aporie est assez complexe et le texte n'en est pas trop clair. Il est utile de distinguer quatre parties : (i) 997 a34-b12, introduction et contestation de la position des Formes; (ii) 997 b12-32, argument contre la position d'intermédiaires, objet des sciences mathématiques et séparés des sensibles; (iii) la portion 997 b32 - 998 a 6 justifie qu'on ait là une aporie car, en réalité, ces sciences ne portent pas sur des sensibles; (iv) argument contre la position d'intermédiaires immanents aux sensibles. Si je comprends bien l'argument (ii), il est fondé sur la distinction, par l'adversaire, de la géodésie et de la géométrie comme un couple de sciences analogues mais dont la première porte sur des objets de perception ( $\hat{\omega} \nu$ a $\sigma \theta \alpha \nu o ́ \mu \epsilon \theta \alpha$ ), la

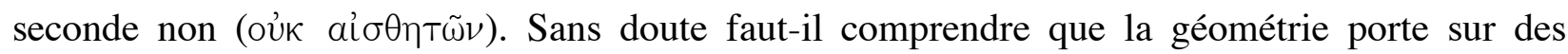
intermédiaires (Aristote ne précise pas s'il s'agit d'intermédiaires ou d'intelligibles). Il ne s'agit donc pas exactement de ce que nous avons dans la classification de Géminus dont le principe de base paraît incompatible avec la position d'intermédiaires séparés des sensibles. Comme ils admettent aussi que l'astronomie, l'harmonie, l'optique - mais aussi la médecine - portent sur des objets de perception, il devrait exister des sciences intermédiaires correspondantes (au même

\footnotetext{
70 Ces deux savants sont associés aux débuts de la mécanique mathématique. C'est sans doute pour cette raison que la tradition fait d'Eudoxe un disciple d'Archytas. V. Diogène Laërce, Vies, VIII, $§ 86$ (qui dit rapporter l'information à partir des Pinakes de Callimaque).

${ }^{71}$ In Euclidem I, ed. Friedlein, p. 77, 1. 15-p. 78, 1. 20. Il dit également que Ménechme fut auditeur d'Eudoxe et qu'il fréquenta Platon (Ibid., p. 67, 1.9-10).

72 V. Met. B, 997 a34-998 a19.
} 
niveau que la géométrie) et donc des "perceptions intermédiaires", des "vivants intermédiaires", une "santé intermédiaire"..., ce qui, pour Aristote, est absurde.

La réfutation est menée sur l'exemple de l'astronomie et du "ciel" intermédiaires : le supposer immobile est impossible (alors ce ne serait certainement pas l'objet de l'astronomie), non plus que de le supposer en mouvement. En effet, auparavant ${ }^{73}$, Aristote avait rappelé que les intermédiaires diffèrent des Formes en ce qu'ils ne sont pas uniques, et des sensibles en ce qu'ils sont éternels et immobiles ! La critique du Stagirite n'ébranle pas vraiment l'idée qu'il y a des sciences portant seulement sur des intermédiaires, la géométrie par exemple, et la portion (iii) ne vise pas non plus cette position. Elle confirme plutôt l'idée que les seuls objets de perception ne suffisent pas à justifier certaines sciences mathématiques. Bref, la position visée par le Stagirite est très proche de celle que nous trouvons chez Géminus, à ceci près que là où celui-ci dit simplement "intelligibles", on dit ici "intermédiaires" ce qui paraît un peu plus sophistiqué et sans doute postérieur.

Il se peut également que l'opposition constitutive ne soit pas envisagée de la même manière. Dans les dialogues, les intelligibles sont présentés comme séparés des objets de perception. Mais on sait, toujours grâce à Aristote, que certains Platoniciens les concevaient comme immanents aux sensibles ${ }^{74}$. Le Stagirite rapproche cette thèse de celle des Pythagoriciens, car il est clair qu'au-delà des difficultés philosophiques qu'elle doit affronter, une position de ce genre est assez "naturelle" pour qui veut garantir l'applicabilité des mathématiques aux choses perceptibles. Or, parmi ses tenants, il cite Eudoxe de Cnide ${ }^{75}$. Malheureusement Proclus présente la classification de Géminus à sa manière - néoplatonicienne - , laquelle ne permet pas de trancher entre les deux possibilités concernant la relation intelligibles-sensibles : séparation ou immanence. Il me paraît donc difficile d'aller plus loin. Reste que l'élaboration philosophique du

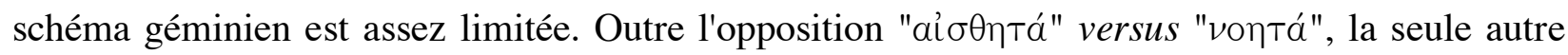

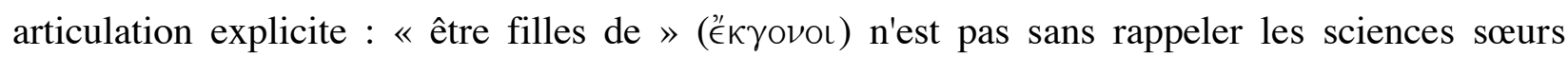
pythagoriciennes. Cette façon simple d'élargir la famille suggère que l'on est en présence d'un schéma proposé par des mathématiciens avant tout soucieux de proposer une description plus complète que le quadrivium.

Si le Stagirite a circonscrit la mathématique par rapport à la physique et à la théologie, aucun texte du corpus n'est explicitement consacré à la classification des espèces de ladite science. Cela dit, différents critères de comparaison entre savoirs y sont proposés, qui, systématisés, pourraient produire un tel système. Ainsi le plus universel l'emporte sur le plus particulier, le plus difficile sur le plus facile. On peut ordonner les sciences en fonction de leur connaissance plus ou moins exacte des causes ou par leur degré d'exactitude. On jugera

\footnotetext{
73 V. Met, A, 6, 987 b14-19.

74 V. Met. A, 9, 991 a14-19.

75 A noter qu'ici, dans (iv) il ne s'agit pas d'intelligibles immanents aux choses perceptibles, mais d'intermédiaires. Aristote n'identifie pas sa cible. Eudoxe avait-il complété sa position après que Platon eut introduit les intermédiaires ? Sont-ce ses disciples ? Ou bien une possibilité envisagée par le seul Aristote lui-même ? Le commentaire d'Alexandre ( $C A G$, I, ed. Hayduck, p. 201, 1. 15sq ) suggère qu'il pourrait s'agir d'Eudoxe, mais il ne l'affirme pas explicitement.
} 
supérieure une étude cultivée pour elle-même plutôt qu'une connaissance poursuivie pour son utilité$^{76}$. Il y a différentes caractérisations de la plus grande exactitude : connaître à la fois le fait et le pourquoi est plus exact que de connaître seulement le fait; une science qui ne s'occupe pas

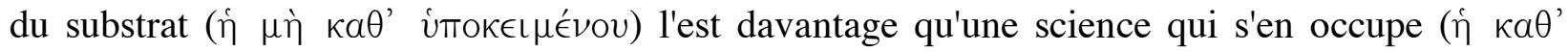

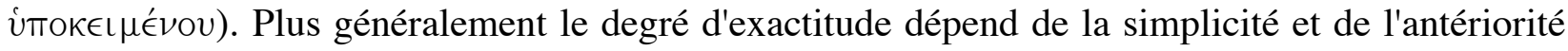
logique de l'objet d'étude. Ce qui procède à partir de principes moins nombreux ( $\dot{\eta} \epsilon^{\epsilon} \xi$

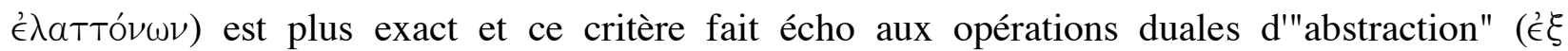

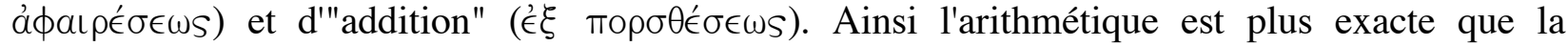
géométrie. Ces deux-là sont plus exactes que les sciences qui considèrent le mouvement et parmi ces dernières, celle qui étudie le premier mouvement, l'astronomie, est la plus exacte ${ }^{77} \ldots$

Mais le critère d'articulation le plus spécifiquement aristotélicien est celui qui articule les

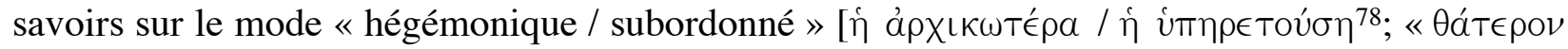

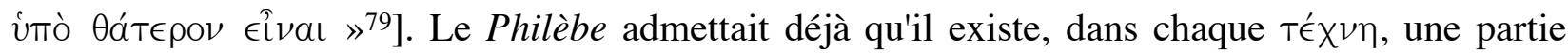
hégémonique, selon une métaphore en quelque sorte psychologique. Le calcul correspondait à la partie rationnelle de l'âme. Chez Aristote, la dualité « hégémonique / subordonnée » concerne des sciences distinctes et relève principalement de la théorie de la démonstration. Des différents exemples proposés par le Stagirite ${ }^{80}$, on peut extraire le schéma suivant :

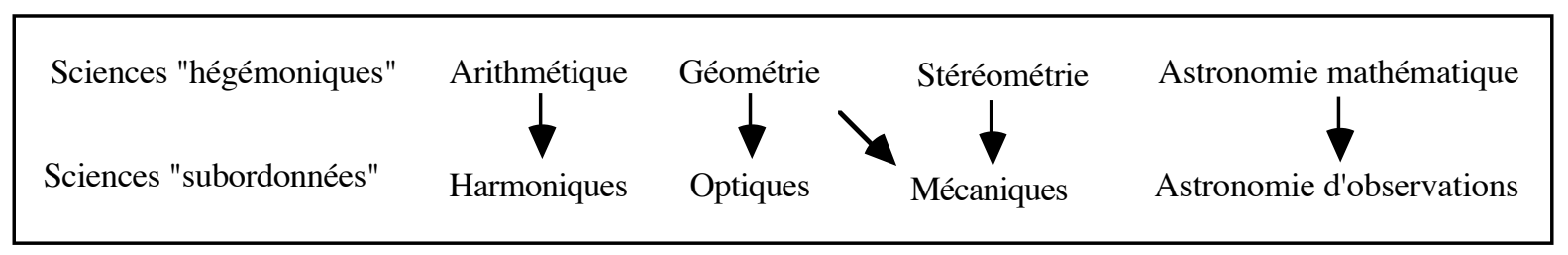

La thématique de la subordination des sciences chez Aristote est assez complexe et réclamerait un examen approfondi qui n'a pas sa place ici ${ }^{81}$. Le schéma ci-dessus n'est pas vraiment comparable à une classification, malgré les éléments communs qu'il comporte avec le système de Géminus. Au demeurant, le Stagirite propose parfois d'autres associations. Ainsi, dans un célébrissime passage de la Physique, il mentionne « les plus physiques des sciences mathématiques » et énumère l'optique, l'harmonique et l'astronomie ${ }^{82}$. Contrairement à Barnes, je ne crois pas que l'on puisse y adjoindre la mécanique, trop marquée du sceau de l'artificialité. Ailleurs, il est dit que l'astronomie est la science mathématique la plus voisine de la philosophie ${ }^{83}$ et l'on doit remarquer que, contrairement à ce qu'affirmeront des auteurs postérieurs, Aristote ne dit jamais que l'astronomie est subordonnée à la géométrie (et à l'arithmétique). Les sciences

\footnotetext{
76 V. Met. A, 2, 982 a8-16 + 25-28.

77 V. par exemple Met. M, 3, 1078 a9-17; An. Post., I, 27, 87 a31-37.

78 V. par ex. Met., A, 2, 982 a 16-17.

${ }^{79}$ V. par ex. An. Post., I, 13, 78 b35-39.

80 V. An. Post., I, 7, 75 b 14-17; I, 9, 76 a9-15 + 22-25; I, 13, 78 b32-79 a16 (l'énumération la plus complète).

${ }^{81}$ V. Aristotle, Posterior Analytics, translated with a commentary by J. Barnes, Oxford, Clarendon Aristotle Series, 1994, pp. 158-162.

82 V. Phys., B, 2, 194 a7-8.

${ }^{83}$ V. Met. $\Lambda, 8,1073$ b4-5
} 
subordonnées aristotéliciennes ne coïncident donc pas avec les disciplines appliquées aux sensibles de la classification de Géminus et nous avons vu, en discutant la quatrième aporie de Métaphysique $\mathrm{B}$, que le Stagirite en contestait même le principe.

Si la "matière" est à peu près identique, le principe de mise en ordre qu'il propose est plus sophistiqué, surtout en ce qui concerne les liens qui unissent les sciences entre elles. A l'articulation verticale que constitue la subordination, on pourrait ajouter la distinction horizontale entre principes propres à une science et principes communs. Ce sont des questions que les classifications ultérieures, notamment médiévales, devront affronter.

Si les éléments essentiels semblent avoir été élaborés dès le $\mathrm{IV}^{\mathrm{e}}$ siècle avant notre ère, au sein de l'Académie et du Lycée, quelle a été la contribution de Géminus ? Celle-ci s'est sans doute limitée à ajouter un certain nombre d'espèces et de sous-espèces pour tenir compte des développements de l'époque hellénistique. Peut-être est-il responsable de la précision selon laquelle l'extension des mathématiques ne saurait être indéfinie. Certaines additions ne sont pas très heureuses, je pense aux sous-espèces de l'arithmétique. La position de sous-discipline attribuée à la gnomonique ou à la dioptrique, voire à la sphéropée, semble quelque peu arbitraire. Elle traduit sans doute le souci de ne pas modifier la division initiale en six sciences principales appliquées aux sensibles. Certaines spécialités, telle la géographie mathématique, sont absentes. Géminus ne pouvait pourtant pas ignorer les travaux de Dicéarque, Ératosthène et Hipparque, tous antérieurs à lui, vu leur importance et le fait que l'école stoïcienne, à laquelle il appartenait, semble s'être particulièrement intéressée à cette discipline. Pour ma part, j'y vois un indice supplémentaire que les grandes lignes du schéma remontent bien à l'époque de l'Ancienne Académie et d'Aristote.

\section{Conclusion}

Les classifications des sciences mathématiques procèdent de l'activité érudite, voire scolaire, qui se développe à la charnière des périodes hellénistique et impériale. Mais leurs éléments, sous forme de programmes éducatifs, de remarques critiques sur les relations entre disciplines ..., ont été élaborés plus tôt, au IV ${ }^{\mathrm{e}}$ siècle avant notre ère. En prenant de l'altitude on pourrait comparer cette période avec celle de l'Encyclopédie. Cette même époque voit en effet Eudème de Rhodes rédiger les histoires concernant les trois sciences principales (arithmétique, géométrie, astronomie) et, vers la fin de ce même siècle, Euclide compose son "encyclopédie" mathématique dont les matières correspondent assez bien aux subdivisions principales de la classification dite de Géminus.

Suivre la postérité du quadrivium dans l'Antiquité tardive, en particulier dans le néoplatonisme latin, est impossible ici ${ }^{84}$. Son succès a été considérable car, quelle que soit son

\footnotetext{
${ }^{84}$ Sur les classifications des sciences mathématiques chez les philosophes d'expression arabe, en particulier al-Kindî, al-Færæbî et Ibn Sînæ (l'Avicenne des Latins), v. l'article de J. Jolivet dans Histoire des sciences arabes, R. Rashed (dir.), Paris, Editions du Seuil, 1997, vol. 3, pp. 255-270. Ibn Sînæ est apparemment le premier à proposer une réflexion approfondie sur la notion même de classification des sciences (pp. 264-266). Il combine habilement le
} 
origine historique, il a bénéficié de l'immense prestige de Platon, vite associé aux anciens Pythagoriciens. Durant l'Antiquité tardive, cette autorité a assuré le maintien d'un intérêt minimal pour les sciences mathématiques. A l'époque impériale (celle de Nicomaque et Plutarque) le repli sur une position identifiée comme platonicienne a constitué une véritable régression par rapport aux sciences mathématiques de l'époque hellénistique que reprenaient et développaient alors les Alexandrins Héron, Ménélaos, Ptolémée, et Diophante. L'adoption du quadrivium par les médioplatoniciens comporte une autre ambiguïté. D'un côté, elle participait aux traditions savantes issues de l'érudition alexandrine impliquant la gestion intellectuelle d'un patrimoine antérieur, et, à ce titre, nous lui sommes redevables de beaucoup de nos informations. Mais, ce faisant, elle ne favorisait pas l'esprit de recherche qui avait existé en mathématiques aux époques classique et hellénistique et qui était encore perceptible à l'époque impériale. Être mathématicien, c'était essayer de produire des résultats nouveaux, un trait assez moderne, solidaire de l'idée qu'il faut cultiver les mathématiques pour elles-mêmes, et pas seulement pour se livrer à de sublimes et perpétuelles récapitulations afin d'introduire à la lecture des grands corpus philosophiques.

quadrivium et la subordination aristotélicienne pour obtenir un tableau complet des disciplines mathématiques développées en Grèce ancienne, auxquelles il adjoint l'algèbre (subordonnée à la science du nombre (pp. 266-267). 